\title{
Nitric Oxide PLIF Measurements in the Hypersonic Materials Environmental Test System (HYMETS)
}

\author{
Jennifer A. Inman, ${ }^{*}$ Brett F. Bathel, ${ }^{\dagger}$ Craig T. Johansen, ${ }^{\ddagger}$ Paul M. Danehy, ${ }^{\S}$ Stephen B. Jones, ${ }^{* *}$ Jeffrey G. \\ Gragg, ${ }^{\dagger \dagger}$ and Scott C. Splinter \\ NASA Langley Research Center, Hampton VA, 23681-2199
}

Planar laser-induced fluorescence (PLIF) of naturally occurring nitric oxide (NO) has been used to obtain instantaneous flow visualization images, and to make both radial and axial velocity measurements in the HYMETS (Hypersonic Materials Environmental Test System) $400 \mathrm{~kW}$ arc-heated wind tunnel at NASA Langley Research Center. This represents the first application of NO PLIF flow visualization in HYMETS. Results are presented at selected facility run conditions, including some in a simulated Earth atmosphere $(75 \%$ nitrogen, $20 \%$ oxygen, $5 \%$ argon) and others in a simulated Martian atmosphere (71\% carbon dioxide, $24 \%$ nitrogen, $5 \%$ argon), for specific bulk enthalpies ranging from 6.5 $\mathrm{MJ} / \mathrm{kg}$ to $18.4 \mathrm{MJ} / \mathrm{kg}$. Flow visualization images reveal the presence of large scale unsteady flow structures, and indicate nitric oxide fluorescence signal over more than $70 \%$ of the core flow for specific bulk enthalpies below about $11 \mathrm{MJ} / \mathrm{kg}$, but over less than $10 \%$ of the core flow for specific bulk enthalpies above about $16 \mathrm{MJ} / \mathrm{kg}$. Axial velocimetry was performed using molecular tagging velocimetry (MTV). Axial velocities of about $3 \mathrm{~km} / \mathrm{s}$ were measured along the centerline. Radial velocimetry was performed by scanning the wavelength of the narrowband laser and analyzing the resulting Doppler shift. Radial velocities of $\pm 0.5 \mathrm{~km} / \mathrm{s}$ were measured.

\footnotetext{
* Research Scientist, Advanced Sensing and Optical Measurement Branch, MS 493, AIAA Member.

$\dagger$ Graduate Student, University of Virginia and NASA Graduate Co-op, AIAA Student Member.

* Post-Doctoral Researcher, National Institute of Aerospace; Currently Asst. Prof., University of Calgary, Calgary, Canada.

${ }^{\S}$ Research Scientist, Advanced Sensing and Optical Measurement Branch, MS 493, AIAA Associate Fellow.

*** Research Technician, Advanced Sensing and Optical Measurement Branch, MS 493.

${ }^{\dagger}$ Research Technician, Structures Experiments Branch, MS 188D.

\# Aerospace Engineer, Structural Mechanics and Concepts Branch, MS 190, AIAA Member. 


\section{Introduction}

rcjet facilities are a vital tool in the testing and characterization of materials intended for hypersonic vehicles,
including those designed for planetary entry. Arcjets are capable of producing flows of a higher enthalpy than traditional wind tunnels, and are thus better suited for evaluating and characterizing candidate materials for thermal protection systems. Like other types of hypersonic facilities, arcjets cannot perfectly simulate all the flow conditions relevant to hypersonic flight. For example, significant dissociation of freestream gases typically occurs in arcjets. Determining how to interpret arcjet test results and then extrapolate those results to flight conditions can therefore be complicated. To that end, measurements of the flow conditions of an arcjet facility are needed to validate computational tools to allow for reliable comparisons between simulations and test results. This allows for reliable predictions of aerodynamic parameters and material response in flight environments that cannot be adequately simulated in ground test facilities. Currently, the flow parameters that can be measured conventionally (or calculated from measured quantities) in the Hypersonic Material Environmental Test System (HYMETS) facility at NASA Langley include stagnation pressure; heat flux (semi-catalytic hot-wall, fully-catalytic cold-wall, and/or noncatalytic cold-wall); gas mass flow rates; sonic, stagnation, and bulk specific enthalpy; and arc current, voltage, and power [1]. Nonintrusive measurements of additional flow parameters are therefore desired. An arcjet flow presents a challenging environment for making measurements as the flow is typically characterized as high-enthalpy, low pressure, chemically reacting, and in nonequilibrium. Several non-intrusive techniques have been applied at other arcjet facilities. Diode laser absorption has been demonstrated for making simultaneous velocity and temperature measurements of an argon arcjet plume [2]. Oxygen $(\mathrm{O})$-atom and nitrogen $(\mathrm{N})$-atom laser-induced fluorescence (LIF) have been used to provide temperature, velocity and species concentration (number density) measurements at a single point or along a line[3-5]. Nitric oxide (NO) and O-atom LIF previously have been used in arcjets to measure translational temperature of $\mathrm{O}$ and rotational temperature of NO [6,7]. Additional descriptions of techniques that have been used to make nonintrusive measurements in arcjets can be found in Refs. [8] and [9].

Nonintrusive diagnostics are being implemented in HYMETS to obtain 1) flow visualization information, including measurements of shock standoff distance and flow uniformity assessments, 2) axial and radial velocity measurements in the freestream and near the test sample, 3) species detection and concentration measurements, including both species produced by the arcjet itself and gaseous species resulting from the ablation of test samples, and 4) measurements of rotational, vibrational, and electronic temperature. This paper presents results of the first 
application of NO PLIF flow visualization in an arcjet, as well as the first non-intrusive measurements of radial and axial velocity in HYMETS and progress to varying degrees towards all four of the aforementioned goals. Using NO PLIF, we have demonstrated that flow visualization, and radial and axial velocimetry can be performed over the full flowfield downstream of the nozzle exit for certain ranges of facility test conditions. The measurements will ultimately provide an improved understanding of the operation of the arcjet and will also provide for facility-tofacility and facility-to-flight scalings for materials tests.

\section{Experimental Methods}

\section{A. HYMETS Arcjet Facility}

The HYMETS facility is powered by a $400 \mathrm{~kW}$ supply. When compared to other, larger arc-heated wind tunnel facilities, significant advantages of HYMETS include the relatively small workforce required to operate the facility (typically one technician), long run times (up to several hours), short down-time between runs and sample changes (less than one hour), and relatively low operating costs. Also, numerous optical ports offer a variety of views of the flow and test specimens. Figure 1 shows an overhead schematic view of the arc plasma generator, nozzle, and test chamber. Note that the schematic shows the retracted position of two flow probes, with dashed lines indicating the injected position of one of the probes. (The facility has four such probes, described in more detail below. When looking down the flow axis from the nozzle toward the diffuser, the probes are located at the $45^{\circ}, 135^{\circ}, 225^{\circ}$, and $315^{\circ}$ positions with respect to the horizontal.) This schematic depicts the position of the seven access ports, each of which can be fitted with a $51 \mathrm{~mm}$ ( 2 inch) diameter UV-grade fused silica (quartz) window to provide optical access at wavelengths down to about $180 \mathrm{~nm}$ in the ultraviolet. Six ports are in the horizontal plane, one is below the centerline injected-probe position, and two are above the horizontal plane. Of the six ports in the horizontal plane, four are angled at approximately $45^{\circ}$ to the flow axis and two are angled at $90^{\circ}$ to the flow axis, just downstream of the leading edge of an injected probe. The laser sheet (shown in purple) enters the test chamber through one of the viewing ports. A periscope (two mirrors, indicated by thick black lines) inside the test chamber then directs the laser sheet to the flow. Dashed lines indicate the position of one of the probes when injected into the flow.

A segmented-constrictor direct-current electric arc-heater serves as an arc plasma generator. The slightly diverging flow issues from a convergent-divergent 8 degree half-angle Mach 5 conical copper nozzle with a 12.7 $\mathrm{mm}(0.5 \mathrm{inch})$ diameter throat and a $63.5 \mathrm{~mm}(2.5 \mathrm{inch})$ diameter exit. Process gases consist of nitrogen $\left(\mathrm{N}_{2}\right)$, 
oxygen $\left(\mathrm{O}_{2}\right)$, carbon dioxide $\left(\mathrm{CO}_{2}\right)$, and argon $(\mathrm{Ar})$. Test gasses are injected tangentially into the bore of the arc plasma generator at six discrete locations, where they are heated by a high-voltage electric-arc maintained between the cathode and anode to create a high temperature ionized plasma flow. The electric-arc is spin-stabilized in the arc plasma generator by the vortex motion of the injected test gasses. The test gasses used in the arc plasma generator are supplied by several compressed gas cylinders and can be custom mixed to any desired atmosphere composition. Adjustable volume percentages of $\mathrm{N}_{2}$ and Ar are used as shield gasses near the cathode and anode, respectively, to protect the electrodes from rapid oxidation.

The plasma flow from the arc plasma generator is accelerated through the nozzle and exhausted into a $0.6 \mathrm{~m}$ ( 2 $\mathrm{ft}$ ) diameter by $0.9 \mathrm{~m}(3 \mathrm{ft})$ long vacuum test chamber where it stagnates on one of four water-cooled specimen/instrumentation injection stings arranged symmetrically around the inside circumference of the test chamber. The flow is then captured by a collector cone with a $0.2 \mathrm{~m}$ ( 8 inch) diameter inlet plane, a $0.15 \mathrm{~m}$ (6 inch) diameter constant cross-section diffuser, and a coiled-copper tubing heat exchanger to decelerate and cool the flow. A two-stage, continuous-flow, high-mass-capacity, mechanical pumping system, is used to evacuate the plasma flow from the facility. The whole facility is cooled by a re-circulating chiller with associated booster pumps and heat exchangers.

Four probes can alternately be hydraulically injected into the flow $51 \mathrm{~mm}$ ( 2 inches) downstream of the nozzle exit. Three of these probes typically consist of a pitot tube to measure stagnation pressure, a Gardon gauge and a copper slug calorimeter to measure fully-catalytic cold-wall heat flux. The fourth probe is usually configured as either a Teflon ${ }^{\circledR}$ slug calorimeter to measure non-catalytic cold-wall heat flux, a silicon carbide (SiC) probe to measure semi-catalytic cold-wall heat flux, or a test specimen. For the results presented herein in which a probe was inserted into the flow, the probe used was a $25 \mathrm{~mm}$ diameter $\mathrm{SiC}$ probe.

A more thorough description of the facility, including detailed explanations of the gas injection system, the instrumentation available in the facility, schematics and photographs, comparisons with other similar facilities, and measured free stream quantities across a wide range of flow conditions can be found in [1]. 


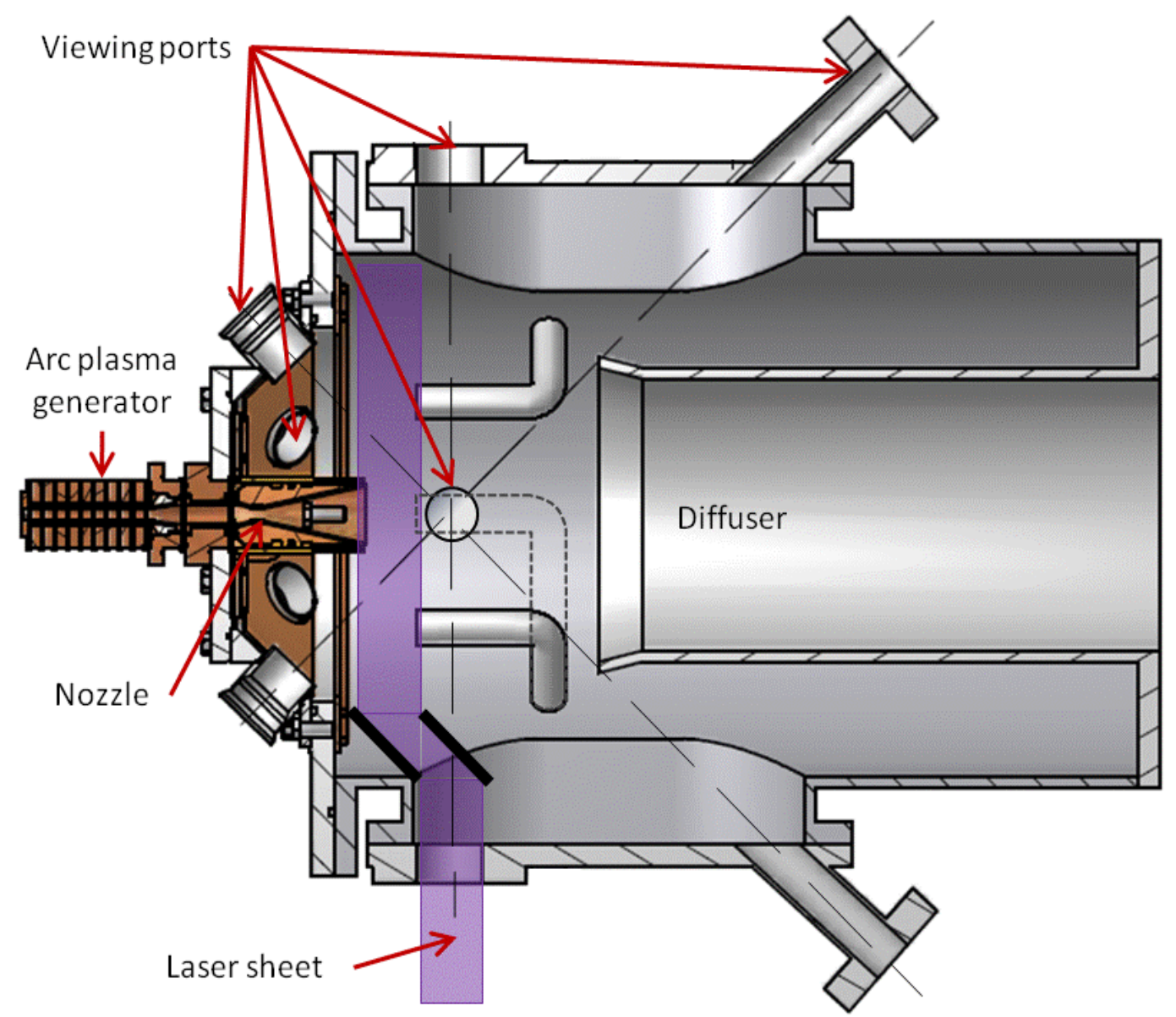

Fig. 1. Schematic of the HYMETS test section.

\section{B. Test Conditions}

Two different gas mixtures were used for the present study. The first is used to simulate atmospheric entry conditions on Earth and consisted of a 75\% nitrogen $\left(\mathrm{N}_{2}\right), 20 \%$ oxygen $\left(\mathrm{O}_{2}\right), 5 \%$ argon $(\mathrm{Ar})$ mixture by volume. The second is used to simulate atmospheric entry conditions on Mars and consisted of a $71 \%$ carbon dioxide $\left(\mathrm{CO}_{2}\right)$, $24 \% \mathrm{~N}_{2}, 5 \%$ Ar mixture by volume. The total mass flow rate was varied from 76 standard liters per minute (slpm) to 404 slpm. The arc current was varied between $100 \mathrm{~A}$ and $200 \mathrm{~A}$. These run conditions resulted in an arc plenum pressure (upstream of the nozzle) of between $31 \mathrm{kPa}$ and $130 \mathrm{kPa}$, and a specific bulk enthalpy between $6.5 \mathrm{MJ} / \mathrm{kg}$ $(2,790 \mathrm{BTU} / \mathrm{lbm})$ and $18.4 \mathrm{MJ} / \mathrm{kg}(7,910 \mathrm{BTU} / \mathrm{lbm})$. Hereafter, the conditions of a given run will be referenced by the specific bulk enthalpy and by the test gas mixture ("Earth" or "Mars" for short). We estimate an upper bound on the average free stream static translational temperature to be $\sim 1,300 \mathrm{~K}\left(\sim 1,900^{\circ} \mathrm{F}\right)$ for the $6.5 \mathrm{MJ} / \mathrm{kg}$ Earth condition and $\sim 1,600 \mathrm{~K}\left(\sim 2,400{ }^{\circ} \mathrm{F}\right)$ for the $10.8 \mathrm{MJ} / \mathrm{kg}$ Mars condition. See section III.C.4. for an explanation of how this estimate was obtained. Table 1 contains additional flow parameters for selected runs corresponding to cases for 
which results are presented in this paper. Asterisks indicate estimated or interpolated values based on measured data from similar runs.

Table 1. Test conditions of runs described in this paper.

\begin{tabular}{|c|c|c|c|c|c|c|c|c|}
\hline$\underset{\#}{\text { Run }}$ & $\begin{array}{l}\text { Test } \\
\text { Gas }\end{array}$ & $\begin{array}{c}\text { Bulk } \\
\text { Enthalpy } \\
M J / k g \\
\end{array}$ & $\begin{array}{c}\text { Arc } \\
\text { Current } \\
\text { Amps } \\
\end{array}$ & $\begin{array}{c}\text { Mass } \\
\text { flow } \\
\text { slpm }\end{array}$ & $\begin{array}{c}\text { Arc } \\
\text { Pressure } \\
k P a \\
\end{array}$ & $\begin{array}{c}\text { Chamber } \\
\text { Pressure } \\
k P a \\
\end{array}$ & $\begin{array}{c}\text { Stagnation Probe } \\
\text { Pressure } \\
k P a \\
\end{array}$ & Measurement Type \\
\hline 4 & Earth & 15.4 & 105 & 102 & 34 & 0.104 & 0.091 & Flow Visualization \\
\hline 5 & Earth & 15.4 & 105 & 102 & 34 & 0.015 & NA & Signal Fraction \\
\hline 9 & Earth & 15.9 & 125 & 128.6 & 44 & $<0.024$ & NA & Signal Fraction \\
\hline 10 & Earth & 18.4 & 150 & 128.6 & 46 & 0.127 & 0.11 & Flow Visualization \\
\hline 11 & Earth & 18.4 & 150 & 128.6 & 46 & $<0.028$ & NA & Signal Fraction \\
\hline 13 & Earth & 16.7 & 150 & 154.6 & 54 & $<0.032$ & NA & Signal Fraction \\
\hline 15 & Earth & 15.5 & 151 & 178.5 & 62 & $<0.044$ & NA & Signal Fraction \\
\hline 17 & Earth & 14.7 & 150 & 203 & 69 & $<0.031$ & NA & Signal Fraction \\
\hline 19 & Earth & 13.7 & 150 & 228 & 77 & $<0.029$ & NA & Signal Fraction \\
\hline 21 & Earth & 12.8 & 150 & 254 & 84 & $<0.033$ & NA & Signal Fraction \\
\hline 22 & Earth & 12.8 & 150 & 254 & 84 & 0.025 & NA & Signal Fraction \\
\hline 24 & Earth & 12.0 & 150 & 280 & 91 & 0.028 & NA & Signal Fraction \\
\hline 25 & Earth & 11.4 & 150 & 304 & 98 & 0.189 & 0.18 & Flow Visualization \\
\hline 26 & Earth & 11.4 & 150 & 304 & 98 & 0.031 & NA & Signal Fraction \\
\hline 29 & Earth & 15.2 & 200 & 304 & 107 & 0.043 & NA & Signal Fraction \\
\hline 35 & Earth & 10.9 & 174 & 404 & 130 & 0.041 & NA & Signal Fraction \\
\hline 37 & Earth & 8.0 & 125 & 404 & 117 & 0.220 & 0.21 & Flow Visualization \\
\hline 39 & Earth & 8.0 & 125 & 404 & 117 & 0.039 & NA & Signal Fraction \\
\hline 44 & Earth & 6.5 & 100 & 403 & 109 & 0.039 & NA & Signal Fraction \\
\hline 77 & Mars & $14.0 *$ & 100 & 100 & $38^{*}$ & $0.079 *$ & $1.90^{*}$ & Flow Visualization \\
\hline 80 & Mars & 15.5 & 100 & 76 & 31 & 0.073 & 1.62 & Flow Visualization \\
\hline 81 & Mars & 10.8 & 100 & 152 & 53 & 0.093 & 2.51 & Flow Vis and Sig Frac \\
\hline 85 & Mars & 10.8 & 100 & 152 & 53 & 0.093 & 2.51 & Radial Velocity \\
\hline 114 & Earth & 6.5 & 100 & 400 & 109 & 0.228 & 0.21 & Radial Velocity \\
\hline 157 & Earth & 6.5 & 100 & 400 & 109 & 0.228 & 0.21 & Axial Velocity \\
\hline
\end{tabular}

\section{NO PLIF Flow Visualization}

The PLIF laser system includes a tunable Nd:YAG-pumped dye laser with a Rhodamine dye mixture followed by a mixing crystal. Optics formed the beam into a laser sheet $\sim 50 \mathrm{~mm}$ wide by $\sim 0.2 \mathrm{~mm}$ thick (FWHM) in the measurement region. The laser sheet was oriented in the horizontal plane relative to the laboratory frame of reference and perpendicular to the axis of the primary flow. Fluorescence was imaged through the optical access port on the bottom of the test chamber, onto a gated, intensified CCD at a viewing angle approximately normal to the laser sheet. Images were acquired at $10 \mathrm{~Hz}$ with a $1 \mu$ s camera gate.

The laser was tuned to the $\mathrm{N}=13$ line of the $\mathrm{Q}_{1}$ branch near $225.7053 \mathrm{~nm}$. (In this notation, "N" is the rotational quantum number of the state probed by the laser and "Q" indicates a set of transitions for which the change in rotational quantum number between the probed state and the laser-excited state is zero. The subscript 1 indicates that the parity of both the upper and lower states is positive, meaning that in both states, the electronic spin is aligned 6 American Institute of Aeronautics and Astronautics 
with the total angular momentum of the molecule.) While the fluorescence signal levels were comparable for the more commonly used $\mathrm{N}=1-3$ lines, $\mathrm{N}=13$ is a well-isolated line, which is desirable for obtaining a good Dopplershift velocity measurement.

For most arc jet conditions, we acquired 100 single shots with the sample injected. If arc jet conditions permitted, we also acquired some images without the sample in order to look at the core flow of the arc jet. If the sample is left out of the flow for too long the cooling lines in the diffuser are damaged, so obtaining images of the core flow with no sample was not possible at some conditions.

Difficulties with the placement of optics (in particular, two mirrors which form a periscope inside the test section, shown in Fig. 1) resulted in a laser sheet which did not quite reach to the nozzle exit on the upstream edge of the laser sheet (although diffuse scatter off the nozzle is visible in the images, if the contrast is adjusted). This is an area for improvement in later tests, and in fact was improved for the quantitative velocity measurements shown below. The downstream edge of the laser sheet skimmed the face of the sample. Vertically, the horizontallyoriented laser sheet was aligned with the center of the flow. The laser sheet was fairly uniform in intensity, although a few striations are noticeable in the images.

The camera (a Princeton Instruments PIMAX-II intensified 512x512 pixel CCD camera) is effectively looking up through a round window port in the bottom of the test section. Since this port is directly below the sample and since the desired field of view is upstream of the sample, the camera is looking back toward the nozzle at an angle. The intensifier gate width was set to $1 \mu$ s with a constant gain of 250 .

\section{Molecular Tagging Velocimetry (MTV) for Axial Velocity Measurements}

The NO PLIF MTV method involves writing a pattern of lines into the flowfield and observing these lines at two different times. The displacement of the lines is used determine the flow velocity component perpendicular to the lines. To form a laser sheet, the collimated $226 \mathrm{~nm}$ beam was passed through a cylindrical lens, which focused and then diverged the beam, expanding it in one direction while leaving it collimated in the other. A spherical lens then collimated the diverging axis of the beam and focused the other axis into a thin sheet approximately $60 \mathrm{~mm}$ wide by 0.5-mm thick. To tag multiple lines of NO in the test section for a velocimetry measurement, a $50 \mathrm{~mm}$ long, LaserOptik GmbH diffusion welded lens array of $25,1 \mathrm{~m}$ focal length cylindrical lenses focused the laser sheet into 
25 lines, running parallel to the model surface in the spanwise direction. The spacing of the lines was approximately $2 \mathrm{~mm}$.

To image the tagged lines, a Cooke DiCAM-PRO camera, with an intensified 1280x1024 pixel array interline progressive scan CCD, was used. The camera used a $100 \mathrm{~mm}$ focal length, F/2.0 Bernhard Halle Nachfolger GmbH lens. When used in double shutter mode, the camera is capable of acquiring an image pair with a minimum $500 \mathrm{~ns}$ delay between the end of the first gate and the beginning of the second. Each gate has a minimum duration of $20 \mathrm{~ns}$, with delay settings and durations set in increments of $20 \mathrm{~ns}$. A detailed discussion of a timing sequence methodology similar to the one used in this NO PLIF experiment is provided in Refs. [10] and [11]. The magnification of the images (pixels $/ \mathrm{mm}$ ) factors directly into the measurement of the velocity, and into the error analysis. To determine the magnification accurately, images were acquired of a planar surface imprinted with a regular pattern of dots that was placed in the same plane as the laser sheet. Use of this so-called dotcard allowed perspective distortions to be corrected as well [10].

\section{E. Doppler Velocimetry for Radial Velocity Measurements}

Doppler-shift-based PLIF velocimetry is an established measurement technique and has been demonstrated on various supersonic and hypersonic flow applications [12-16]. The Doppler effect shifts the location of the spectral line center relative to the static vacuum center if the flow has a velocity component in the direction of the laser. This Doppler shift, and thus the flow velocity, can be determined from NO PLIF imaging in a variety of ways, including both fixed- and tunable-frequency methods. Fixed-laser-frequency measurement schemes can measure velocity instantaneously and so are preferred when time-resolved velocity measurements are required. The process of scanning the laser frequency limits this method to measuring time-averaged velocity. Tuned-frequency schemes, however, are less susceptible to systematic error [17] and the dynamic range of the technique is not limited by the finite width of the spectral line or laser line as is with fixed-frequency schemes [18]. In the current study, Dopplershift based velocimetry was used to obtain quantitative distributions of radial velocity for two flow conditions simulating a $10.8 \mathrm{MJ} / \mathrm{kg}$ Mars atmosphere (Run 114) and 6.5 MJ/kg Earth atmosphere (Run 85). By scanning the laser over a small wavelength range, the excitation spectrum of the relatively well-isolated $\mathrm{Q}_{1}(13)$ transition of NO was captured on a series of images using the Princeton Instruments PIMAX-2 intensified CCD camera with $512 \mathrm{x}$ 512 pixel resolution. Specifically, the scan ranges were $3.5 \mathrm{pm}\left(\lambda_{L}=225.7075-225.7040 \mathrm{~nm}\right)$ with a scan step size of $0.05 \mathrm{pm}$ and 10 images per wavelength for the $10.8 \mathrm{MJ} / \mathrm{kg}$ Mars case (a total of 720 images acquired over 72 
seconds); and $4.4 \mathrm{pm}\left(\lambda_{L}=225.7079-225.7038 \mathrm{~nm}\right)$ with a scan step size of $0.1 \mathrm{pm}$ and 10 images per wavelength for the $6.5 \mathrm{MJ} / \mathrm{kg}$ Earth case (a total of 450 images acquired over 45 seconds). Post-analysis of these images revealed a shift in the excitation spectra, which was used to determine absolute magnitudes of radial velocity.

\section{Analysis and Results}

\section{A. Flow Visualization Results}

\section{Single-Shot Images}

Figures 2, 3 and 4 show flow visualization results for selected enthalpy conditions for Earth and Mars, respectively. For each condition, three false-color single-shot images are shown. The flow is from left to right. The nozzle exit is clearly visible on the left side of Fig. 2, and the face of the 1-inch diameter silicon carbide sample is clearly visible on the right side of the images. The intensity in Fig. 2, which is an averaged image, has been adjusted so that reflected light from the nozzle can be seen relative to the luminosity of the sample.

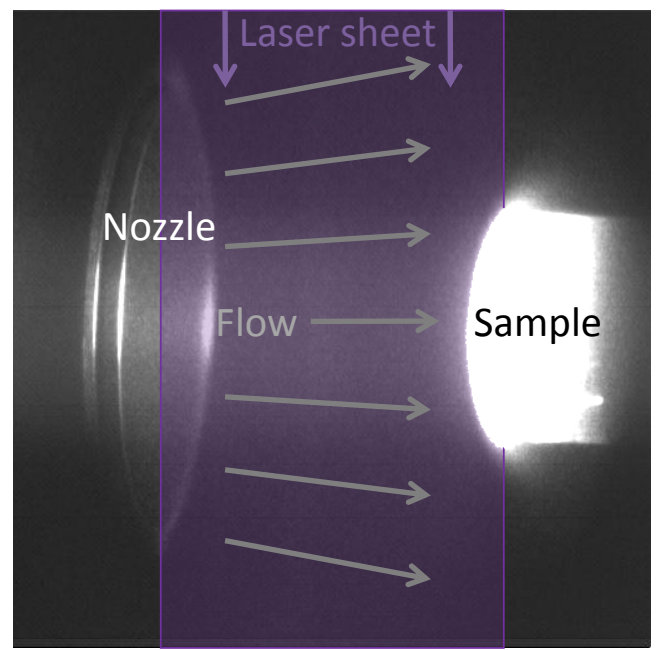

Fig. 2. Location of laser sheet relative to nozzle exit and $\mathrm{SiC}$ probe.

The instantaneous NO PLIF images in Figs. 3 and 4 show highly non-uniform flow containing large-scale flow structures ahead of the bow shock. (Note that false-color maps with arbitrary scaling have been applied. The images in Fig. 4 appear grainier than those in Fig. 3 because the absolute signal intensity was generally lower in the Mars cases than in the Earth cases for comparable enthalpies.) These highly irregular structures appear mushroom-shaped in some images. NO fluorescence was observed over a large percentage of the core jet flow on a shot-to-shot basis at 
the lower enthalpy conditions. As enthalpy was increased, NO fluorescence became more intermittent in the core jet flow. NO fluorescence was observed only on the edges of the jet flow for the highest enthalpy conditions.

One phenomenon that we observed while analyzing data from many runs was the variable nature of the observed luminosity between the bow shock and the surface of the sample. For some runs, the observed intensity in this region was roughly symmetric, relatively constant, and independent of laser wavelength, so we attributed it mainly to natural flow luminosity — probably line emission from different atomic and molecular species in the flow. For other runs, the intensity in this region was highly variable from one image to the next, and showed spatial asymmetries that were anticorrelated with the fluorescence intensity upstream of the bow shock. Further study is warranted to ascertain the nature of the flow in the region between the bow shock and the sample, including the spectral profile of the natural flow luminosity and the dependence of both natural flow luminosity and laser-induced fluorescence on flow enthalpy, composition, laser excitation wavelength, and laser intensity. See section 5 below for a more detailed discussion.

The bow shock on the sample probe is clearly visible in many of the single-shot images, and can be seen in a few of the selected sample images shown in Fig. 3. The shock is evidenced by a sudden decrease in fluorescence in front of the sample probe. As described above, images of dotcards were acquired which allow for the determination of absolute scale in the images. Using an average of 100 single shots from a low-enthalpy air run where the bow shock in front of the stagnation probe was clearly visible (6.5 MJ/kg Earth, Run 36), the shock standoff distance was measured to be $9.5 \pm 1.1 \mathrm{~mm}$. 


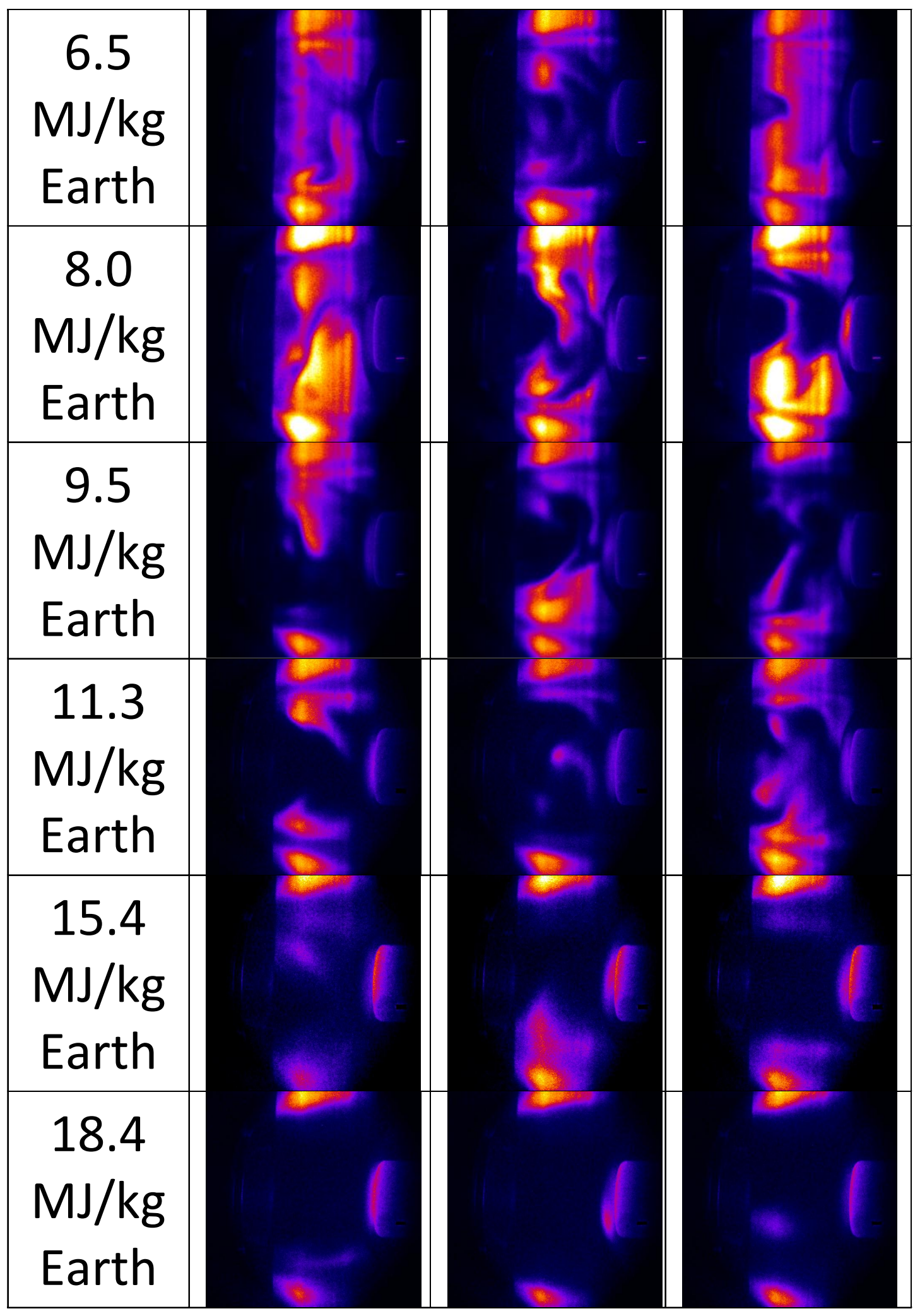

Fig. 3. Single-shot NO PLIF images in false color for Earth atmosphere simulations, at various enthalpies. 


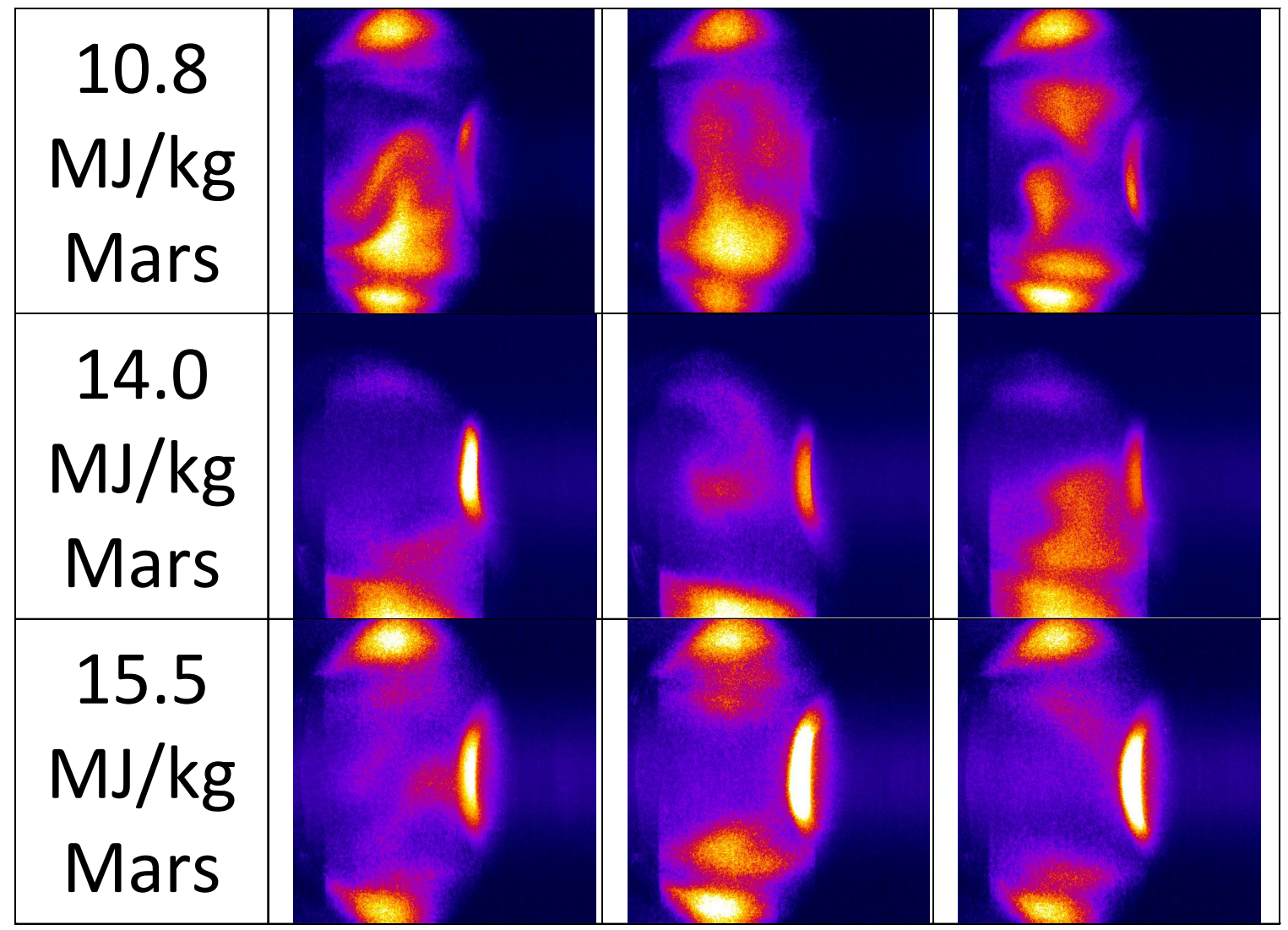

Fig. 4. Single-shot NO PLIF images in false color for Mars atmosphere simulations, at various enthalpies.

\section{Variation in Fluorescence Signal Intensity with Enthalpy}

One approximate indication of the concentration of NO in the flow is the magnitude of the fluorescence signal; while fluorescence intensity depends on many factors (including temperature, pressure, density, species mole fractions/quenching), in general, more fluorescence correlates with a higher concentration of NO. Figure 5 shows measurements of the mean signal intensity in the core region of the flow over a range of enthalpies. The curve is fitted to "Mean Intensity (Air)" data only. In order to calculate the mean signal intensity, averaged images were cropped to exclude all but the core of the flow. The boundaries defining this region of interest are depicted by a white dashed rectangle in Fig. 6. For the Earth runs, the $\mathrm{SiC}$ probe was retracted briefly to allow images to be acquired of the unperturbed free stream arcjet flow. For Mars conditions, the probe was not retracted, and so the images used to calculate signal fraction were of flows with the $\mathrm{SiC}$ probe injected.

Enthalpies below about $14 \mathrm{MJ} / \mathrm{kg}$ produced detectable signal levels, whereas the images obtained at higher enthalpies were relatively noisy. For all conditions examined, the shot-to-shot standard deviation in the mean intensity level was of the same order as the mean intensity. First, the mean intensity value was calculated for each 
pixel in the measurement region. Then, the standard deviation in the intensity at each pixel in the measurement region was calculated. Finally, the mean value of this standard deviation was calculated for all pixels in the measurement region. The standard deviation relative to the mean signal may provide a qualitative indication of the level of unsteadiness or the presence of turbulence in the flow. Note that the absolute signal levels were lower for Mars cases than for Earth cases at the same specific bulk enthalpy. One possible reason could be that the chemical pathways leading to the creation of NO are different for the two gas mixtures, and so the amount of NO produced with the Mars mixture is less than the amount produced with the Earth mixture at an equivalent enthalpy. All of the flow conditions which we examined in this series of tests exhibited unsteady behavior as indicated by the varying non-uniform PLIF signal distributions in the single-shot images.

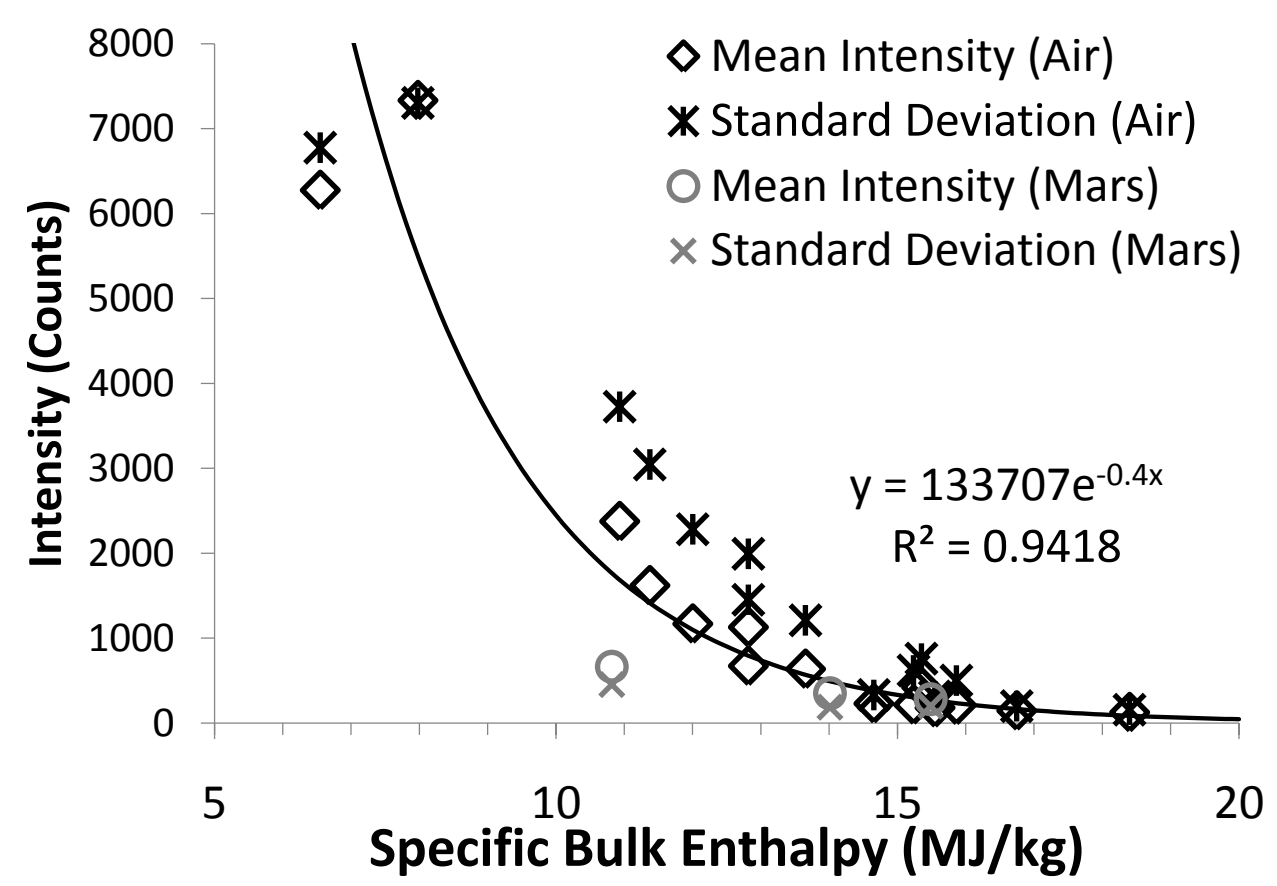

Fig. 5. Measured NO fluorescence intensity versus specific bulk enthalpy. 


\section{Fraction of Core Flow with Fluorescence Signal vs. Enthalpy}

In addition to overall intensity, another metric for the usefulness of NO PLIF as a diagnostic technique in this facility is the percentage of the flow where fluorescence signal is observed. In order to calculate this quantity, hereafter called signal fraction, single-shot images were cropped in the same manner as above, with the boundaries of the measurement region shown in Fig. 6. As in the measurement of mean signal intensity in the previous section,

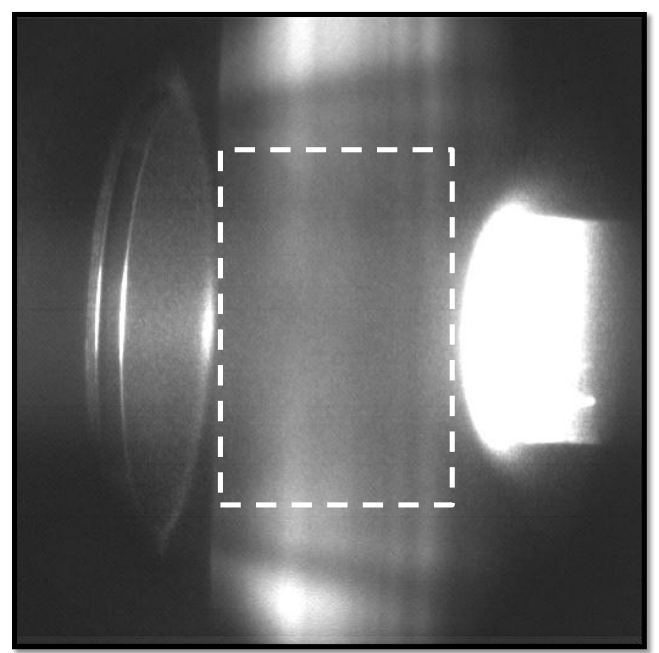

Fig. 6. Indication of relative location and size of measurement region for Figures 7 and 8.

the $\mathrm{SiC}$ probe was retracted for Earth runs but not retracted for Mars runs. After cropping, a uniform background intensity value was subtracted from all the images from a given run. An arbitrary threshold level of 500 counts (about $3 \%$ of the maximum signal intensity obtained) was then applied and the percentage of pixels with an intensity above 500 counts was calculated. 


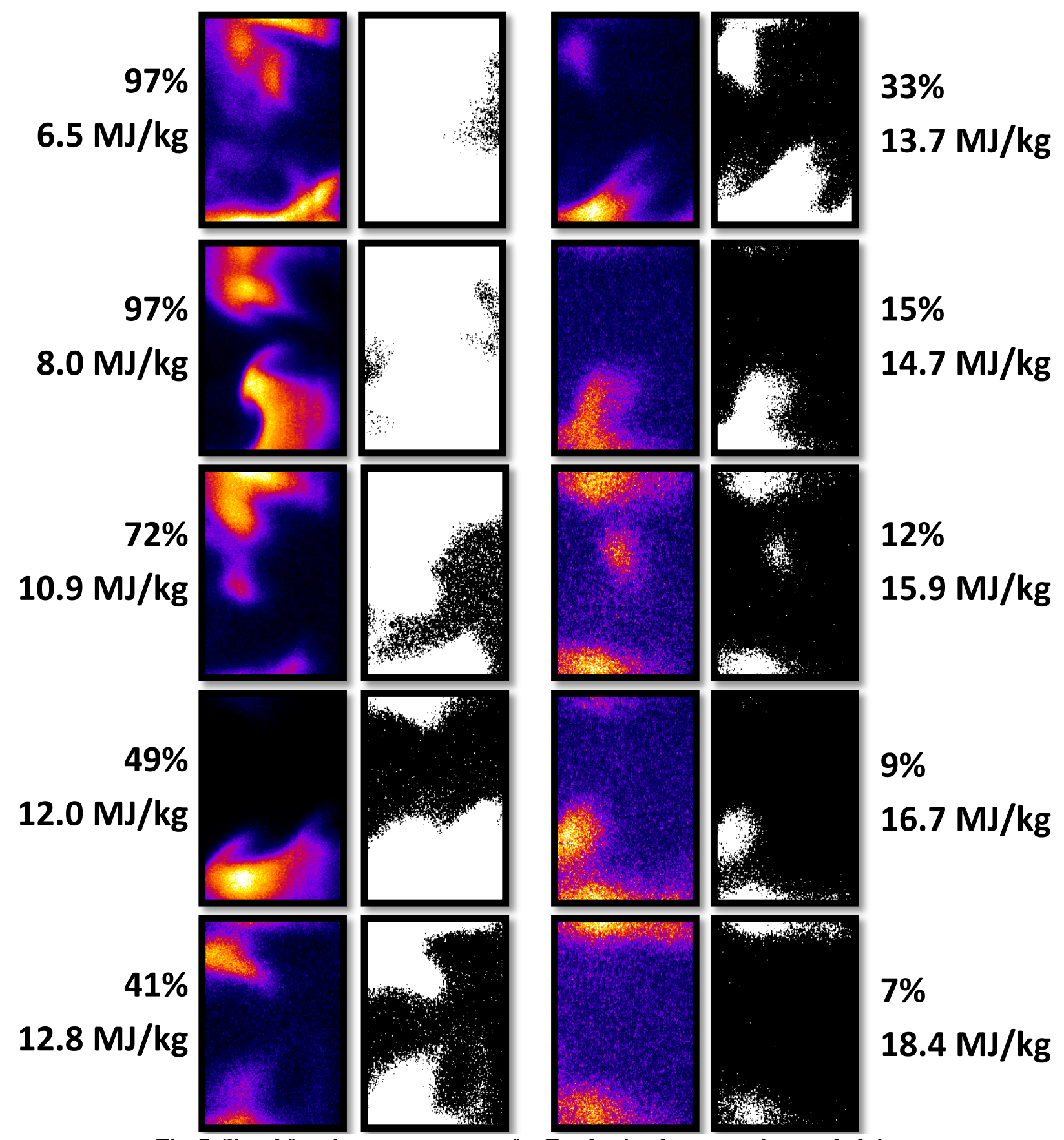

Fig. 7. Signal fraction measurements for Earth stimulant at various enthalpies.

Figure 7 depicts false-color and black and white cropped single-shot images for a range of enthalpies for Earth runs, and Fig. 8 depicts similar images for Mars runs. Percentages indicate the mean proportion of the core flow for each condition with nitric oxide fluorescence signal. The false-color images show a representative single-shot for the given run condition (i.e. the signal fraction in the selected single-shot images is very close to the mean signal fraction for all images in that run). The corresponding black and white images show the effect of background 
subtraction and threshold application, with white pixels representing those with an intensity of greater than the threshold level of 500 counts.

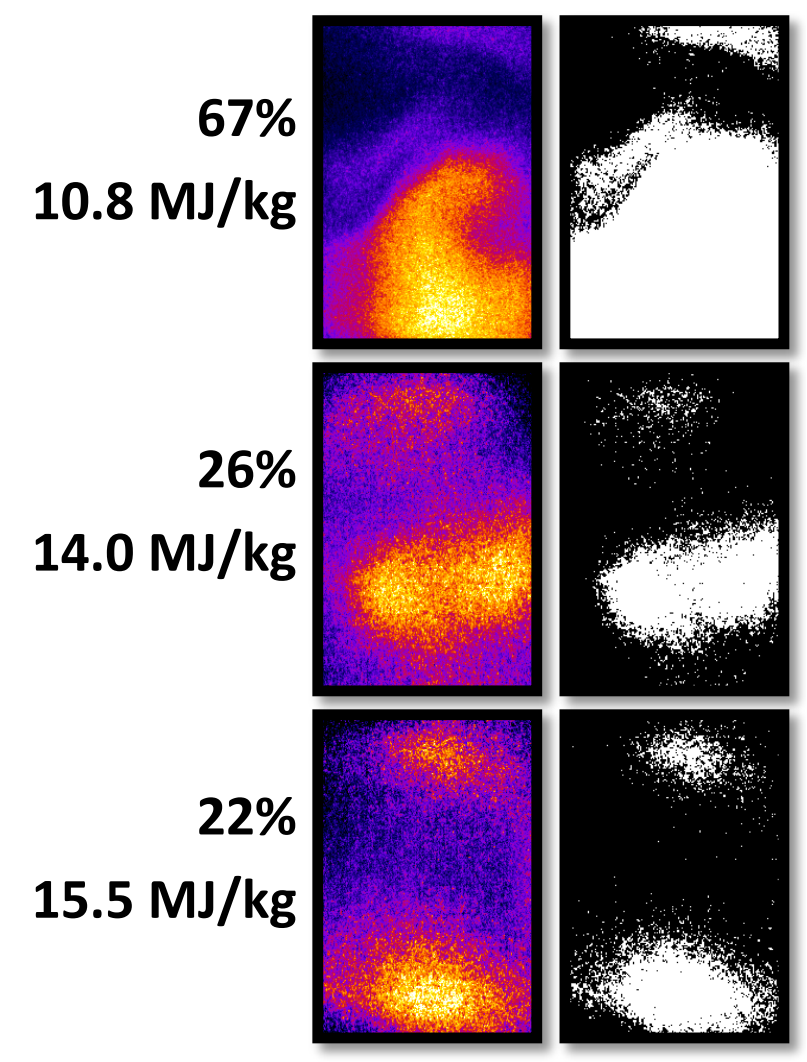

Fig. 8. Signal fraction measurements for Mars stimulant at various enthalpies.

Figure 9 shows a graph of the mean signal fraction as a function of enthalpy. The error bars indicate the standard deviation in the signal fraction for all of the single shot images used to calculate the mean signal fraction for each run. The exponential fitted line was fit to the Earth data; however, the Mars data appears to follow the same trend. Enthalpies below $10 \mathrm{MJ} / \mathrm{kg}$ had signal over almost the entire flow, but over about $12 \mathrm{MJ} / \mathrm{kg}$, less than half of the flow had signal. This provides a guideline for the flow conditions where NO PLIF velocimetry measurements should be feasible, as well as those for which low signal fractions make single-shot molecular tagging velocity measurements unfeasible. This finding was in good agreement with the results of Mizuno et al. who reported that "total enthalpy of under $10 \mathrm{MJ} / \mathrm{kg}$ is suitable" for NO LIF in JAXA's (the Japanese Aerospace Exploration Agency's) $750 \mathrm{~kW}$ arc heated wind tunnel [7]. One additional observation is that for two runs at identical conditions (the two points with an enthalpy of $12.8 \mathrm{MJ} / \mathrm{kg}$ ), two different mean signal fraction values were obtained. The reasons for this are not entirely clear. The difference could be an indication of real variations in the 
arcjet flow, or could perhaps be a byproduct of variations in the probe laser intensity. Future measurements should include simultaneous measurements of laser intensity.

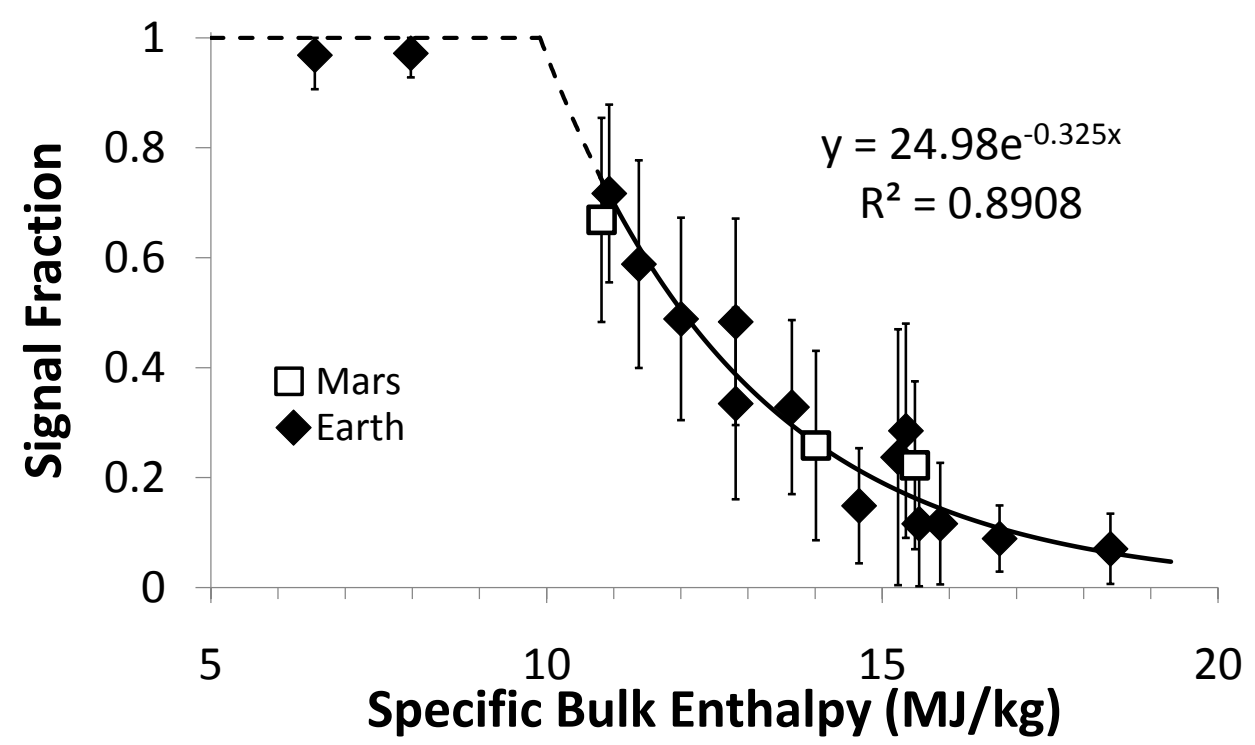

Fig. 9. Fraction of core flow with NO fluorescence signal as a function of specific enthalpy.

\section{Upstream Influence of Stagnation Probe}

An unexpected finding of the flow visualization images was the effect that the presence of a stagnation probe had on the flow upstream of the probe. Figure 10 shows three false-color images from an $8.0 \mathrm{MJ} / \mathrm{kg}$ Earth run. The center image is a composite of the two other images. Note that in all three images, the contrast has been enhanced over the left and right side of the images to show the positioning of the nozzle exit and the $\mathrm{SiC}$ probe (if present). The red image on the left is an average of 42 single shots without a probe in the flow; the green image on the right is an average of 54 single shots from the same run where a $\mathrm{SiC}$ probe is injected into the flow. The center image shows the composite of the two, highlighting the differences between the flow with and without the probe. With the probe in place, the NO PLIF signal extends to the top and bottom of the image. With the probe removed, the NO PLIF is localized closer to the core. In a purely supersonic flow, pressure disturbances cannot propagate upstream. However, in this wind tunnel flow, the subsonic region of the shear layer and the nearly stagnant (perhaps recirculating) flow outside the core of the open jet flow provide mechanisms for pressure disturbances to propagate upstream. Because HYMETS has a diverging conical nozzle, the flow is expected to be slightly diverging as it exits the nozzle. This can be seen in both the averaged image of the unperturbed core flow (i.e. without a probe inserted into the flow) and in the averaged image of the flow with the probe injected. Additionally, there appears to be 
relatively little NO outside the core flow in the no-probe case, but a significant amount of NO fluorescence can be seen in this region in the probe-in case. In the images with the probe inserted into the flow, the angle of divergence appears to be somewhat greater than in the no-probe case. This interpretation is likely misleading because the differences in the images are probably due to different amounts of NO and/or different quenching environments in the shear layer between the core flow and the nearly stagnant flow for the two different conditions. Quenching is a process by which fluorescence intensity is reduced due to collisional energy transfer from excited NO molecules to other molecules. Oxygen is particularly effective at quenching NO fluorescence, so the additional NO present in the stagnant region in the probe-in case may displace ambient oxygen, thereby reducing the amount of quenching in the shear layer.
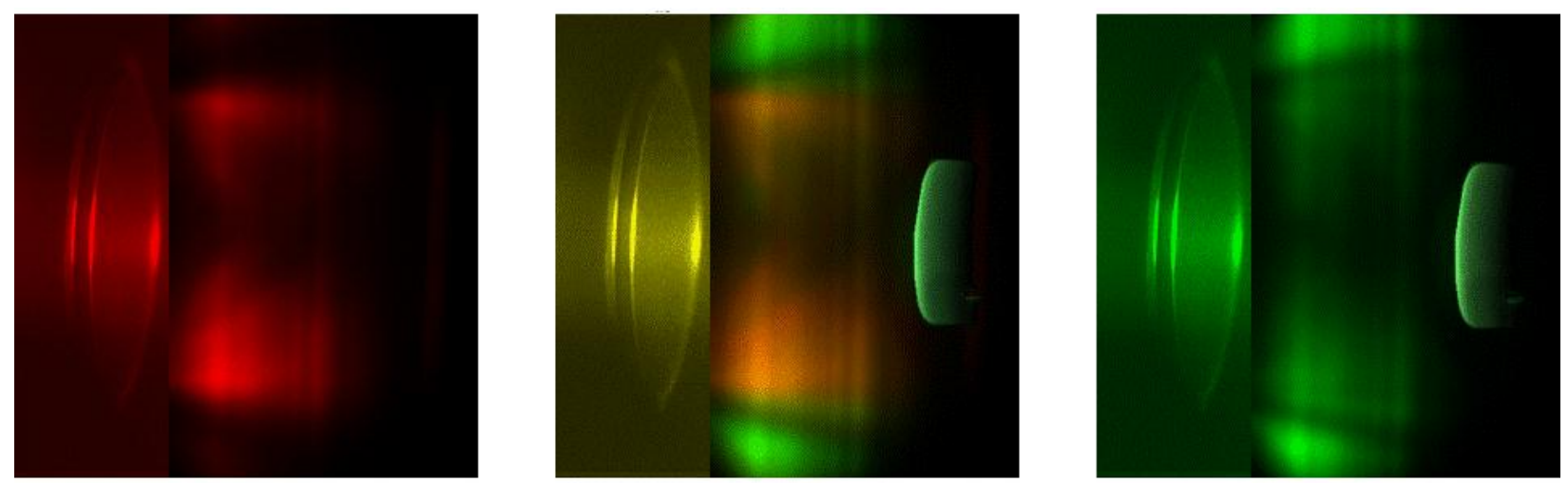

Fig. 10. Comparison of averaged probe-out (left), averaged probe-in (right), and averaged composite (center) false images for Earth simulation.

\section{Correlation of Pre- and Post-shock NO Fluorescence}

Analysis of a sequence of low enthalpy Earth $(6.5 \mathrm{MJ} / \mathrm{kg})$ single-shot images shows a possible anti-correlation between the uniformity of the fluorescence signal upstream of the bow shock and the intensity between the bow shock and the surface of the stagnation probe. In Fig. 11a, the result of averaging all 66 single-shot images from Run 44 is shown. In this image, signal is observed both before and after the bow shock. Figure 11d shows a single-shot image from this set in which the NO fluorescence was non-uniform upstream of the bow shock and relatively moderate signal is observed between the bow shock and the surface of the stagnation probe.

Figure $11 \mathrm{~b}$ displays the average of 11 single-shot images from Run 44 that all showed relatively uniform NO fluorescence upstream of the bow shock. Figure 11d shows a single-shot from this image set. In these images, NO fluorescence is relatively uniform upstream of the bow shock generated by the stagnation probe. Between the bow shock and stagnation probe surface, almost no signal is observed. 
Figure 11c shows the result of averaging 12 single-shot images from Run 44 in which relatively higher intensity was observed betweeen the surface of the stagnation probe and the bow shock. In this figure, a significant decrease in NO fluorescence signal along the centerline flow ahead of the bow shock is observed. Downstream of the bow shock, the signal intensity gradually increases with downstream position, appearing to have a maximum at the surface of the probe. This increased intensity between the bow shock and the probe surface stands in contrast with the signal observed over the same region in Fig. 11b and, to a lesser extent, with Fig. 11a. Figure 11f shows a singleshot from this image set. In this image, the NO fluorescence ahead of the bow shock appears much more intermittent with respect to Figs. 11d and 11e. The signal intensity in this image between the bow shock and the surface of the probe is also higher relative to Figs. $11 \mathrm{~d}$ and $11 \mathrm{e}$.

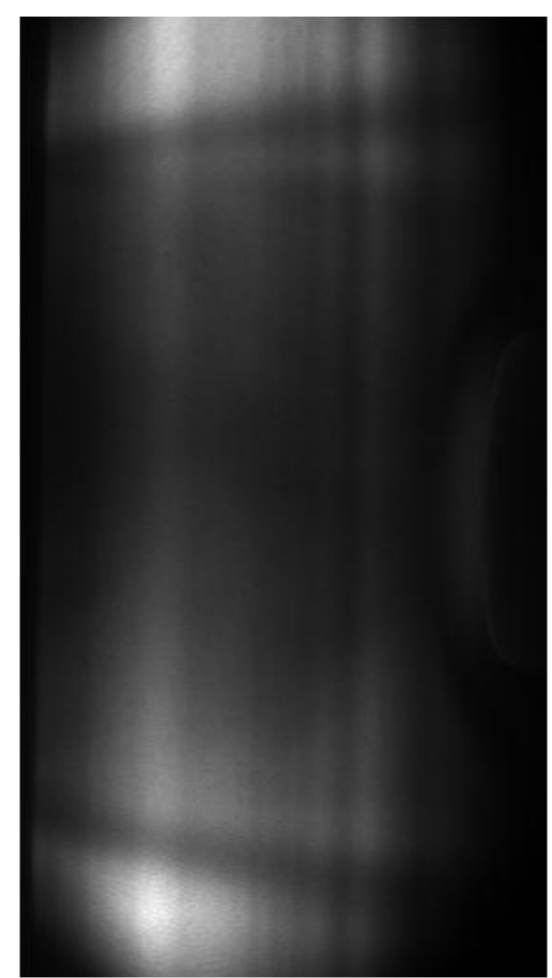

(a)

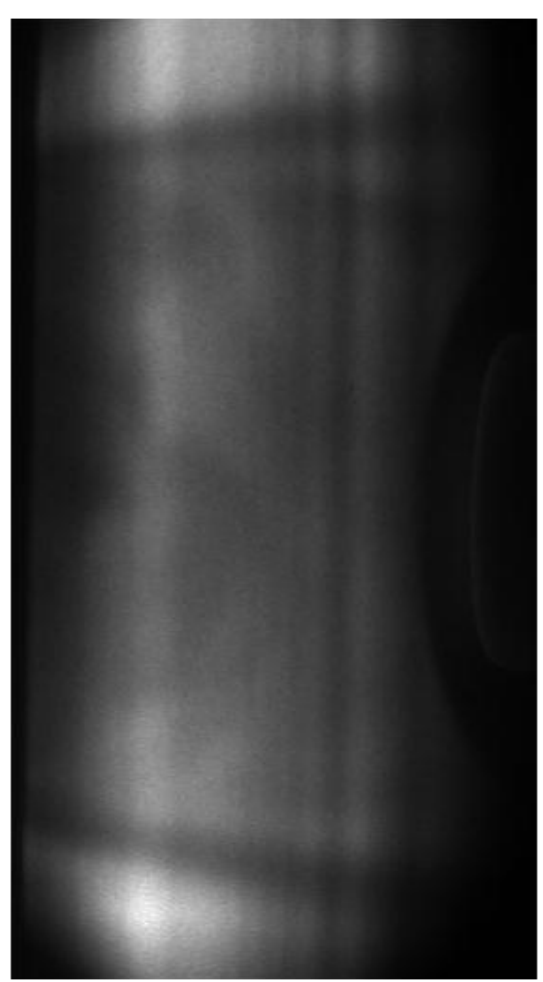

(b)

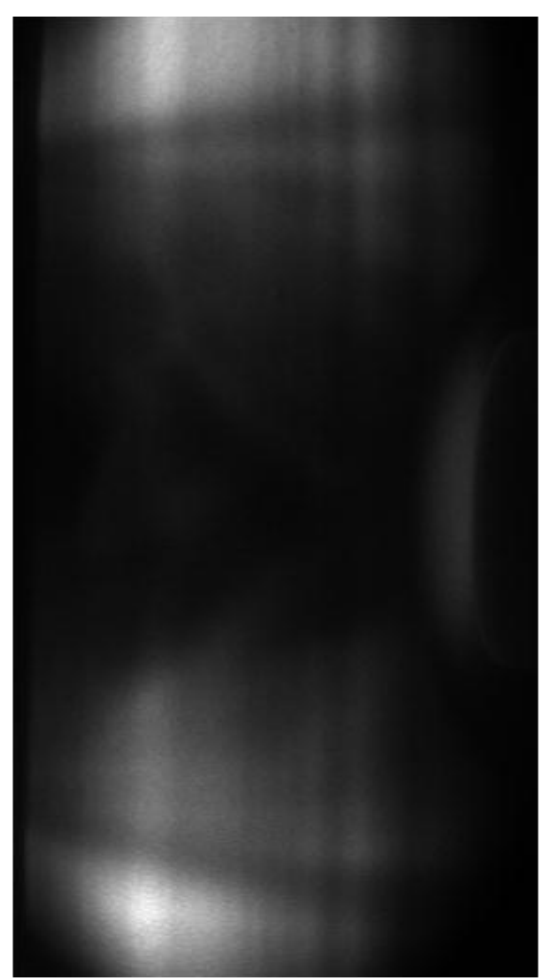

(c) 


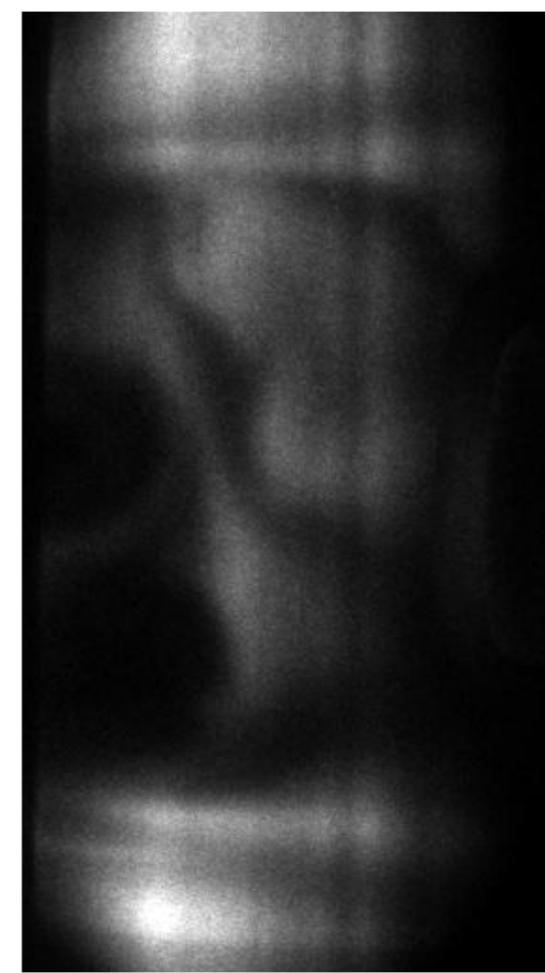

(d)

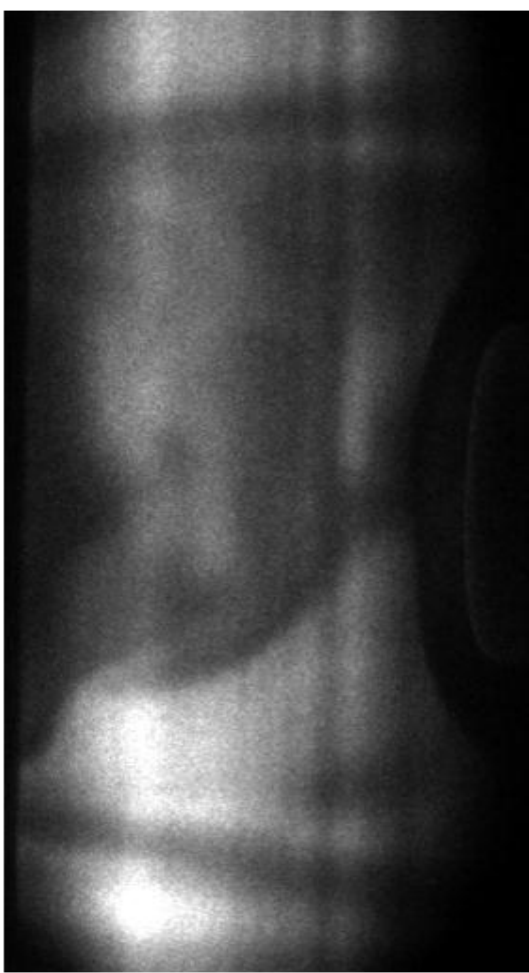

(e)

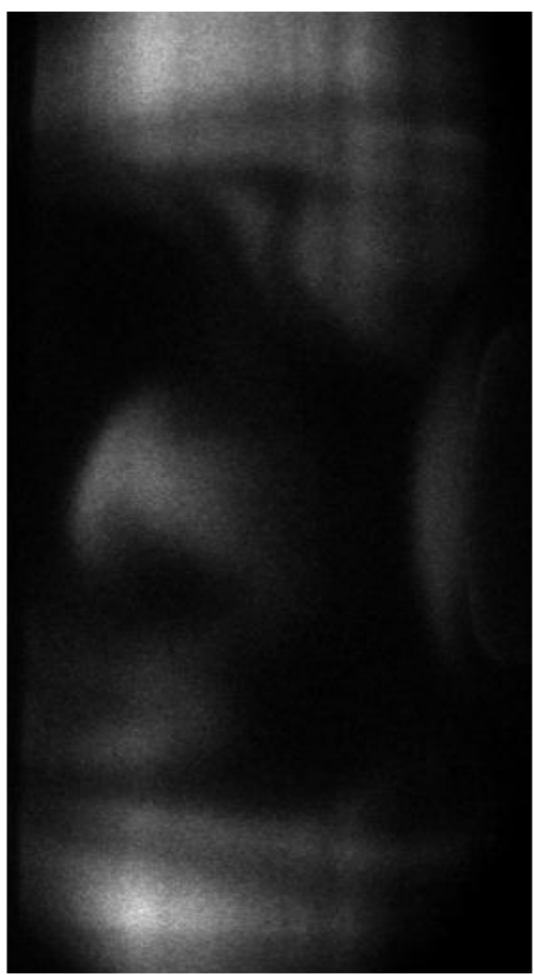

(f)

Fig. 11. Average (top) and single-shot (bottom) comparison of images from Run 44 (6.5 MJ/kg, Earth) with (a, d) uniform NO ahead of the bow shock, $(c, e)$ all images, and $(d, f)$ relatively high signal after the bow shock.

Figure 12 shows the mean signal within a region immediately in front of the bow shock (pre-shock) and plotting it against the mean computed within a region between the bow shock and the surface of the sample (post-shock) on a single-shot basis. The two selected regions are shown in the inset image in Fig. 12. The figure shows that NO fluorescence in the pre-shock region is, to some extent, anti-correlated with the intensity observed in the post-shock region, though the correlation is imperfect. Observation of the single-shot images also shows that the intensity in the post-shock region appears to be aligned with streamlines emanating from pre-shock regions in which NO fluorescence is absent. Additionally, a gradual increase in the post-shock luminosity up to the probe surface was observed in the single-shot images. If the observed intensity is in fact laser-induced fluorescence rather than natural flow luminosity, it would suggest that $\mathrm{NO}$ is being formed in the post-shock stagnation region from cold pre-shock gas that experiences a sharp temperature increase as it is passes through the bow shock. Further study is needed to better understand this observed behavior. 


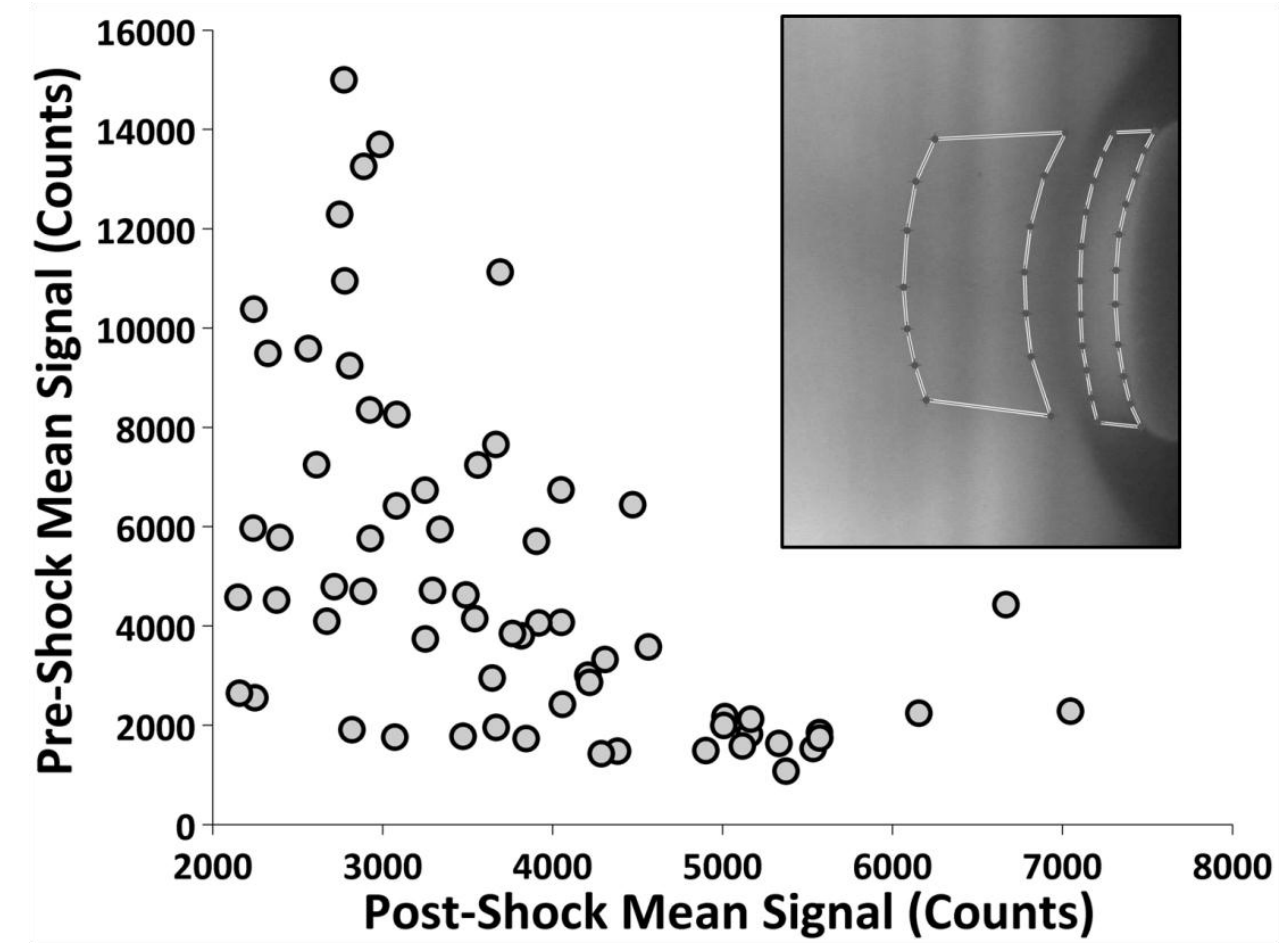

Fig. 12. Relationship between measured mean signal in pre-shock and post-shock regions (positions shown in inset figure).

\section{B. Axial Velocity Analysis and Results}

\section{Axial Velocity Uncertainty Analysis:}

The method used to process both single-shot and average velocities and associated uncertainties is similar to the approach outlined in $[10,11,19]$. However, to investigate the potentially unsteady nature of the core flowfield, the previous method of determining the spatial uncertainty has been modified.

In the previous estimates of single-shot spatial uncertainty, which has been documented in $[10,11]$, the flow is assumed to remain essentially laminar in nature. This assumption led to a formulation of single-shot velocity uncertainty, $u_{V_{X}, \Delta x}$, based upon the standard deviation in the measured single-shot tagged profile shifts, which had been defined as:

$$
u_{V_{X}, \Delta x}=\frac{\partial V_{X}}{\partial(\Delta x)} \cdot u_{\Delta x}=\frac{\partial\left(\frac{\Delta x}{C \cdot \Delta t_{N D}}\right)}{\partial(\Delta x)} \cdot u_{\Delta x}=\frac{1}{C \cdot \Delta t_{N D}} \cdot t_{N-1,95 \%} \cdot \sigma_{\Delta x}
$$

In the above equations, $C$ is the correction factor to the software-specified time separation, $\Delta t_{N D}$, between sequential frames that results in the effective time delay between frames, $\sigma_{\Delta x}$ is the standard deviation of the 21 American Institute of Aeronautics and Astronautics 
observed tagged profile shift in pixels, $t_{N-1,95 \%}$ is the student t-statistic at 95 percent confidence for total number of data points, $N$, at a particular pixel location used to obtain $\sigma_{\Delta x}$, and $u_{\Delta x}$ is the uncertainty in the measured profile shift. For this paper the calculation of the correction factor, which has a dependence on the ratio of peak signal intensities between the initial, $S_{1, \max }$, and delayed, $S_{2, \max }$, frames, can be described by the following relation:

$$
C=a+b\left(\frac{s_{1, \text { max }}}{s_{2, \text { max }}}\right)^{1 / 2}+c \ln \left(\frac{s_{1, \text { max }}}{s_{2, \text { max }}}\right)
$$

with the coefficients $a, b$, and $c$ being $0.987,9.128 \times 10^{-5}$, and $-2.780 \times 10^{-3}$, respectively, for an effective first gate of, $t_{e G 1}$, of $5 \mathrm{~ns}$.

In this paper, we have obtained single-shot spatial measurement uncertainty estimates based on signal-to-noise considerations alone, since the signal-to-noise ratio is the largest contributor to the measurement uncertainty in single-shot measurements. In order to make this estimate, measurements of displacement along several tagged profiles were made in regions well outside of the core nozzle flowfield where the mean axial velocity was measured to be essentially zero for a set of 96 sequential image pairs. Additionally, over the duration of these measurements, the laser was tuned from $226.025 \mathrm{~nm}$ to $226.020 \mathrm{~nm}$ to excite the $\mathrm{Q}_{1}(12)+\mathrm{Q}_{2}(20)$ transition in NO. Tuning the laser away from the absorption peak decreased the PLIF intensity significantly, so a dependence of spatial precision uncertainty on fluorescence intensity could be established. Data points are grouped together in bins according to the maximum intensity measured in the first gate. The bins start with points having peak intensities between 0 and 10 counts and are incremented by 10-count bins up to a maximum of 300 counts. By analyzing the standard deviation of the measured shifts for each bin, the spatial uncertainty is obtained. The calculated spatial uncertainty is then plotted against the average intensity values for each 10-count bin, as shown in Fig. 13. As the signal intensity in the first frame decreases, the measurement uncertainty (and the standard deviation) increases. Based upon these measurements, empirical curve fits of the single-shot standard deviation, $\sigma_{\Delta x\left(S_{1, \max }\right)}$, and uncertainty, $u_{\Delta x}$, as functions of peak signal intensity in the initial frame are obtained:

$$
\sigma_{\Delta x\left(S_{1, \max }\right)}=\left[\alpha_{1}+\beta_{1} \cdot S_{1, \max }\right]^{-1}
$$




$$
u_{\Delta x}=t_{N-1,95 \%} \cdot \sigma_{\Delta x\left(S_{1, \max }\right)}=\left[\alpha_{2}+\beta_{2} \cdot S_{1, \max }\right]^{-1}
$$

with $\alpha_{1}$ and $\beta_{1}$ being $6.327 \times 10^{-1}$ pixels $^{-1}$ and $4.558 \times 10^{-3}$ pixels $^{-1} \cdot \operatorname{counts}^{-1}$, respectively, and $\alpha_{2}$ and $\beta_{2}$ being $3.212 \times 10^{-1}$ pixels $^{-1}$ and $2.297 \times 10^{-3}$ pixels $^{-1} \cdot$ counts $^{-1}$, respectively.

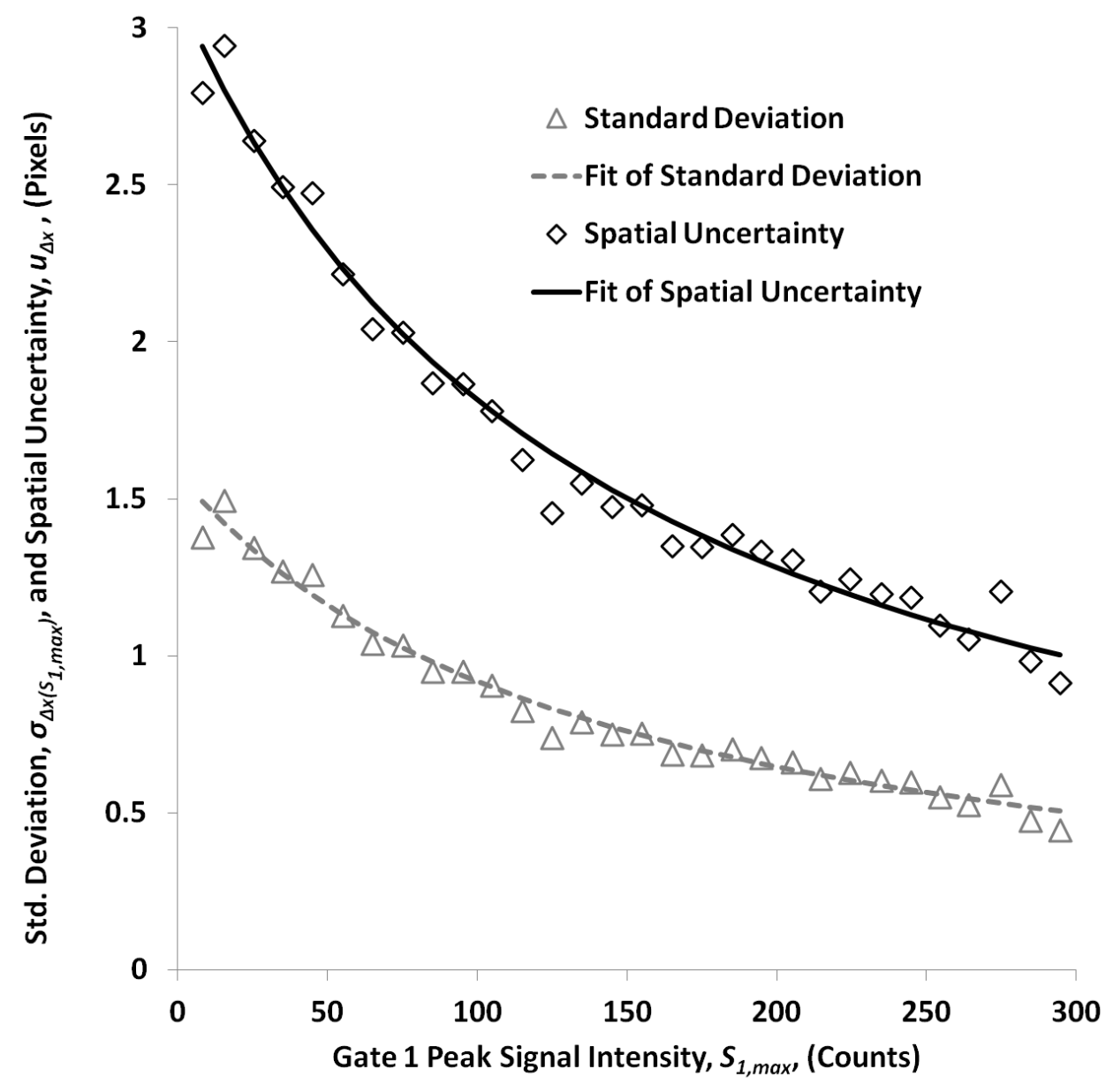

Fig. 13. Determination of spatial uncertainty as a function of signal-to-noise ratio.

To obtain an estimate of the magnitude of fluctuating axial velocity component, $V_{X}{ }^{\prime}$, a relation for the dependence of the standard deviation of the mean velocity, $\sigma_{\overline{V_{X}}}$, must be made that separates out the signal-to-noise dependency identified in Fig. 13. Using the relation for standard deviation of the profile shift precision for singleshot measurements as a function of signal intensity put forth in Eq. (3), the standard deviation of the mean profile shift precision as a function of mean signal intensity is $\sigma_{\overline{\Delta x}\left(\overline{\left.S_{1, \max }\right)}\right.}=\sigma_{\Delta x\left(\overline{\left.S_{1, \max }\right)}\right.}$. Dividing the standard deviation of 
the mean profile shift, $\sigma_{\overline{\Delta x}\left(\overline{\left.S_{1, \max }\right)}\right.}$, through by the corrected time separation between the undelayed and delayed frames, $C \cdot \Delta t_{N D}$, and squaring the result provides the variance in the measured mean velocity due to random fluctuations in the mean signal intensity at the measurement location.

Based upon observations of single-shot velocity distributions throughout each image and the corresponding signal intensities, no apparent correlation between the signal and measured mean velocity magnitude exists. Therefore, the variance in measured mean velocity due to random fluctuations in signal intensity is assumed to be independent of the variance in the measured mean velocity magnitude due to random turbulent fluid mechanical fluctuations. The sum of these two independent variance values results in the total variance (or covariance) in the measured mean axial velocity.

Using the signal-dependent velocity variance relation from Eq. 3 and the total variance in the measured mean

axial velocity, $\left(\sigma_{\overline{V_{X}}}\right)^{2}$, the standard deviation of the axial velocity component due to fluid mechanical fluctuations is:

$$
V_{X}^{\prime}=\sqrt{\left(\sigma_{\overline{V_{X}}}\right)^{2}-\left(\frac{\sigma_{\overline{\Delta x}\left(\overline{S_{1, \max x}}\right.}^{C \cdot \Delta t_{N D}}}{2}\right.}
$$

\section{Image Pre-Processing:}

The spatial resolution for the axial velocity experiments was measured by acquiring an image of a matrix of square marks separated at equal spatial intervals, known as a dotcard, mentioned above and detailed in [20]. To correct for optical and perspective distortion of the images in these experiments, the image of the dotcard in the test section was acquired with the cameras and a corresponding undistorted image of the same dotcard was created with Adobe Acrobat software. An image registration algorithm, UnwarpJ, was then used to correct for distortion [21]. This software is a plug-in created for the image processing software, ImageJ, a freeware image processing program available from National Institutes of Health. ${ }^{\S \S}$ For the axial velocity measurements, once the undistorted axial velocity images were obtained, an undistorted background image, acquired in the absence of any fluorescence, was subtracted from the set.

\footnotetext{
${ }^{\S}$ Software available at http://rsb.info.nih.gov/ij [version 1.43 retrieved January 2010]. 24 American Institute of Aeronautics and Astronautics
} 
To improve the signal-to-noise ratio, MATLAB ${ }^{\circledR}$ was used to bin the images by 16 pixels in the radial direction. This improved the signal-to-noise by consolidating the signal along profiles tagged by the laser. However, the spatial resolution of the measurement was degraded by a factor of 16 in the spanwise direction because of this processing.

\section{Axial Velocity Processing:}

The processing of velocity data is similar to that outlined in [10]. A 1-dimensional cross-correlation method was used to calculate both averaged and instantaneous velocity from the displacement of single profiles. In this paper, no estimations of the radial velocity contribution to uncertainty in the axial velocity measurement have been made. Due to the reduced spatial resolution of this experiment, the tagged profiles appear more closely spaced, 25 pixels peakto-peak, than in previous analyses $[10,19]$. Additionally, the axial velocity magnitudes encountered in the current test are about three times larger than in prior work, resulting in relatively large observed shifts in the profiles between the initial and delayed frames. This presents a difficulty in automating the cross-correlation-based velocity measurement algorithm: a correlation window fixed about the tagged profile, with the same width as the peak-topeak profile separation in the initial frame, will occasionally result in erroneous correlations with neighboring profiles. To avoid this potential error, an initial estimate of the profile shift is provided to the processing software. Prior to processing the data, the initial shift estimate at each point along a given profile is additionally shifted by 2 pixels towards the mean of the all the shift estimates along the respective profile. This is done in order to help identify any correlations which are not consistent with the overall distribution of axial velocities along any one profile. A data rejection threshold that estimated the quality of the cross-correlation coefficient data returned by the measurement software was also used to limit erroneous velocity data. In fitting the data, the root-mean- square (RMS) of the $\mathrm{R}^{2}$ value from a $2^{\text {nd }}$-order polynomial fit to the peak of the normalized cross-correlation coefficients and the peak value of the normalized cross-correlation coefficients were computed at each measurement location. Any points with RMS values below a threshold of 0.95 were rejected in this study. An additional analysis was performed in which a cross-correlation of 2 adjacent profiles was obtained in an attempt to further improve the velocity results. This resulted in a reduction of $10-20 \%$ in uncertainty of the mean velocity compared to the single profile method. However, by using the 2-profile correlation method, there is a tradeoff made between the reduced 
uncertainty and axial spatial resolution, which is reduced by a factor of 2 . Therefore, in this paper only results from the single-profile correlation method are presented.

\section{Axial Velocity Results:}

Figures 14a and 14b show averaged undelayed and delayed images, respectively. These images were obtained by averaging the 240 pre-processed individual sequential image pairs used to compute both single-shot and mean axial velocities for a $400 \mathrm{slpm}$ flow with a $100 \mathrm{~A}$ arc current applied (6.5 MJ/kg Earth). The effective time separation between the two images is $550 \mathrm{~ns}$. Each line is approximately 13 pixels wide, and typical displacements along the centerline are approximately 20 to 21 pixels. The sample is seen to the right side of the images.

Figure 15a shows the mean axial velocities measured along the $4^{\text {th }}, 11^{\text {th }}$, and $16^{\text {th }}$ tagged lines. The center of the black data points correspond to the measured mean velocities and the widths of these points correspond to two times the associated uncertainty of the mean velocity at each measurement location. The portions of these profiles lying outside of the core flowfield have noticeably smaller (near zero) mean velocities and uncertainties, relative to the core flow region downstream of the nozzle. Along the $11^{\text {th }}$ profile located $1.97 \mathrm{~cm}$ downstream of the exit plane of the nozzle, the mean velocity outside the core flow is approximately $5 \mathrm{~m} / \mathrm{s}$ with an uncertainty of $16 \mathrm{~m} / \mathrm{s}$. This relatively low uncertainty is attributed to the increased signal-to-noise levels, persistence of signal throughout all of the sequential image pairs, and negligible fluid mechanical unsteadiness relative to the core nozzle flow. Within the core of the flow, an increase in both mean velocity and uncertainty is observed. For this same profile, located 1.97 $\mathrm{cm}$ downstream of the nozzle exit plane, the mean velocity and uncertainty within a $1-\mathrm{cm}$ region centered about the axis of symmetry are $3,035 \mathrm{~m} / \mathrm{s}$ and $59 \mathrm{~m} / \mathrm{s}$, respectively. Figure $15 \mathrm{a}$ also provides the fluctuating velocity component, $V_{X}{ }^{\prime}$, computed from Eq. (5), and represented by the open circle data points. For this same 1-cm region about the centerline along the $11^{\text {th }}$ profile, the mean value of the standard deviation, $V_{X}{ }^{\prime}$, is $243 \mathrm{~m} / \mathrm{s}$. This represents velocity fluctuations that are approximately $8.0 \%$ of the mean velocity. 


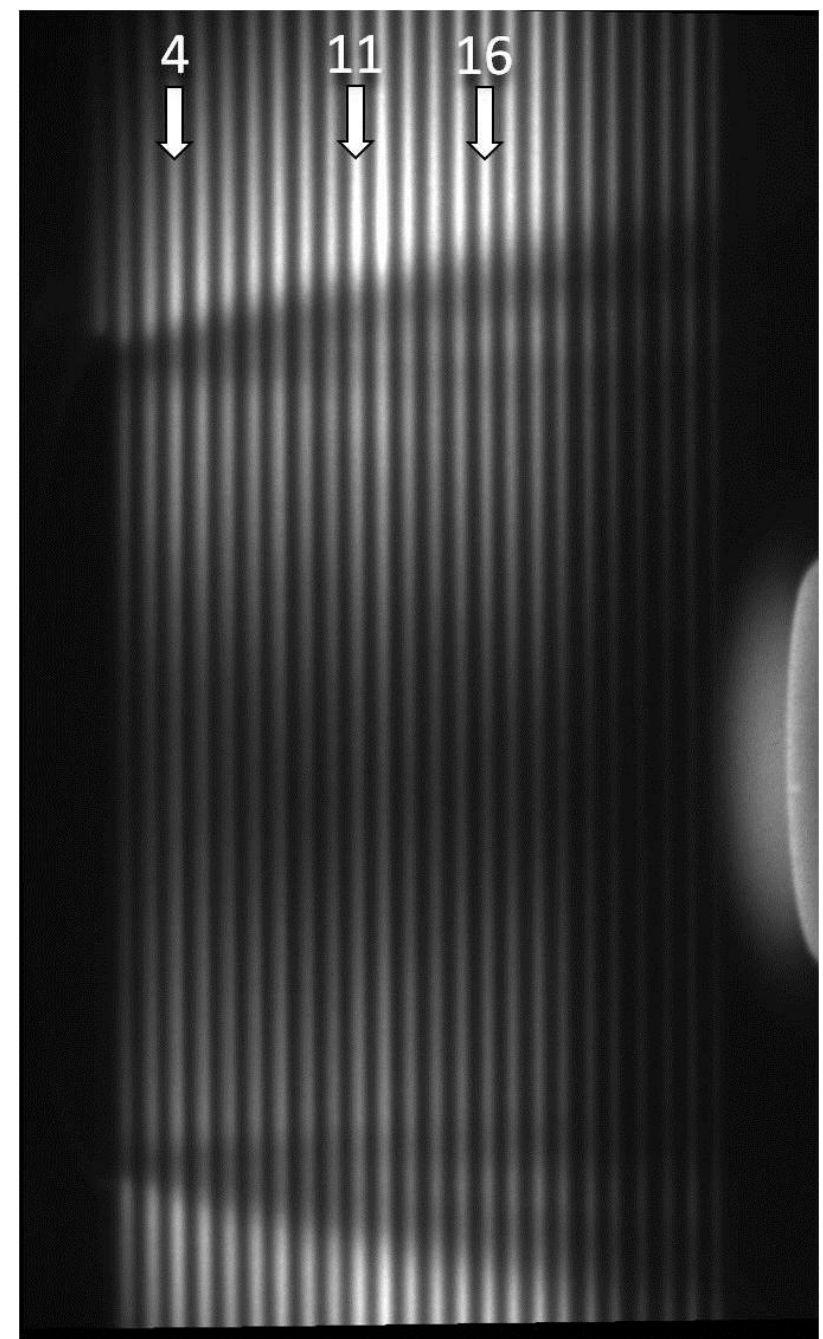

(a)

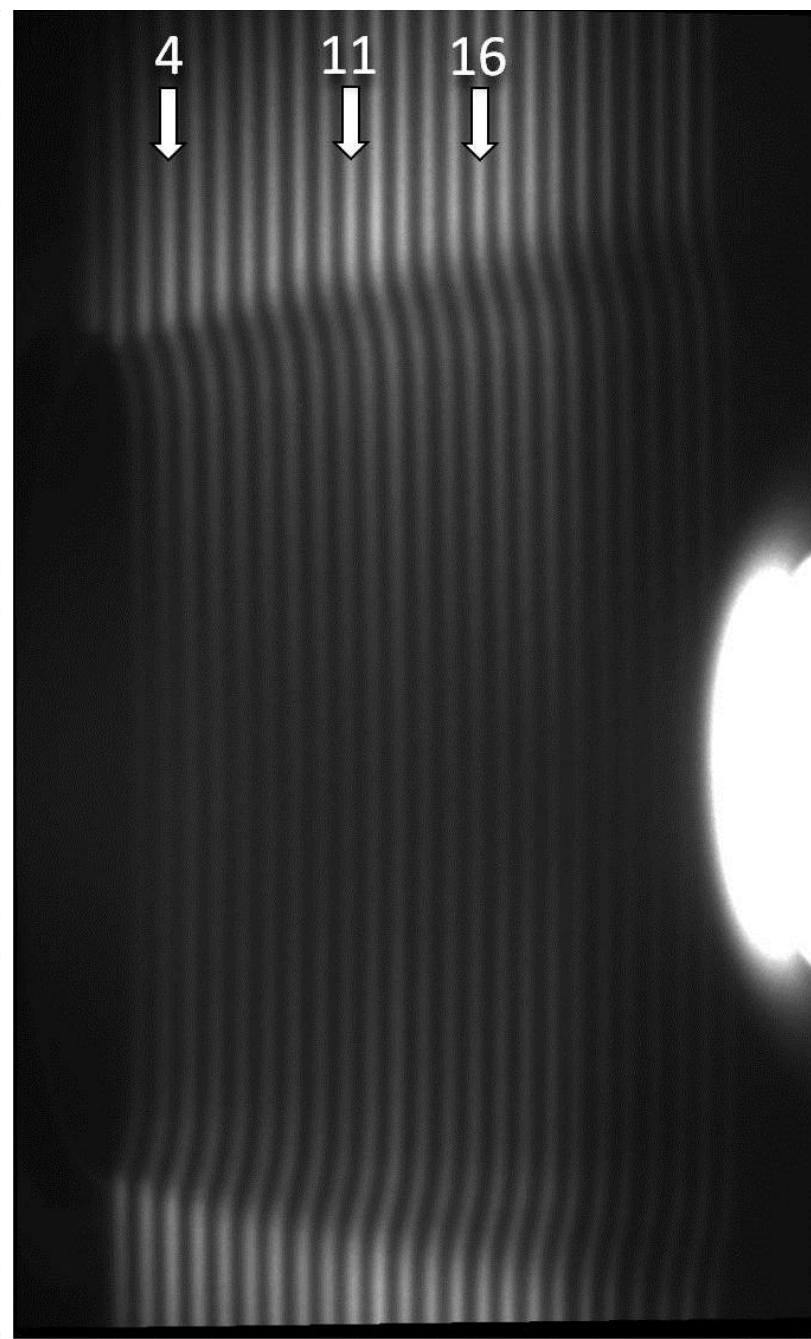

(b)

Fig. 14. Pre-processed average image from (a) undelayed and (b) delayed frame obtained during axial velocity measurements.

Figure $15 \mathrm{~b}$ provides a set of single-shot velocity measurement data along the same profile regions shown in Fig. 15a. While the single-shot profiles shown in Fig. $15 \mathrm{~b}$ are well populated with data points, the intermittent presence of NO and reduced signal levels in the core of the nozzle flow often result in single-shot velocity profiles that are sparsely populated with data. Typically within the core nozzle flow, only 10 to 70 percent of the total image pairs at a particular pixel location yield a measureable shift. Outside of the core of the nozzle flow, the approximate yield increases to greater than 85 percent. 


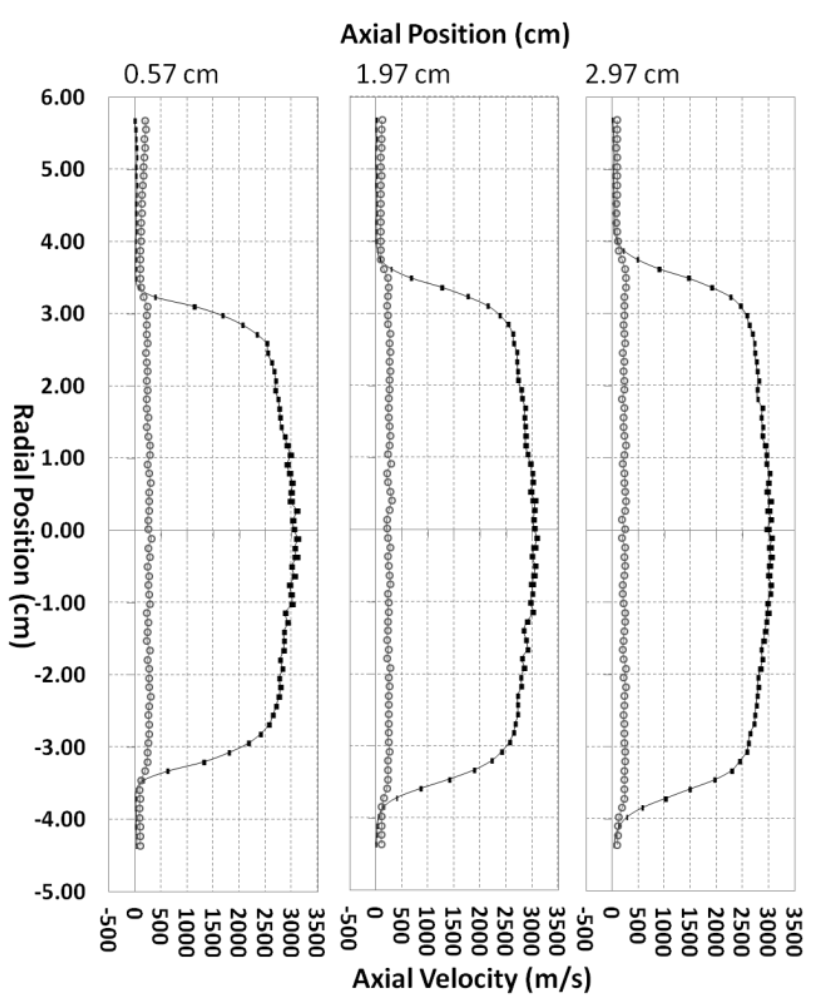

(a)

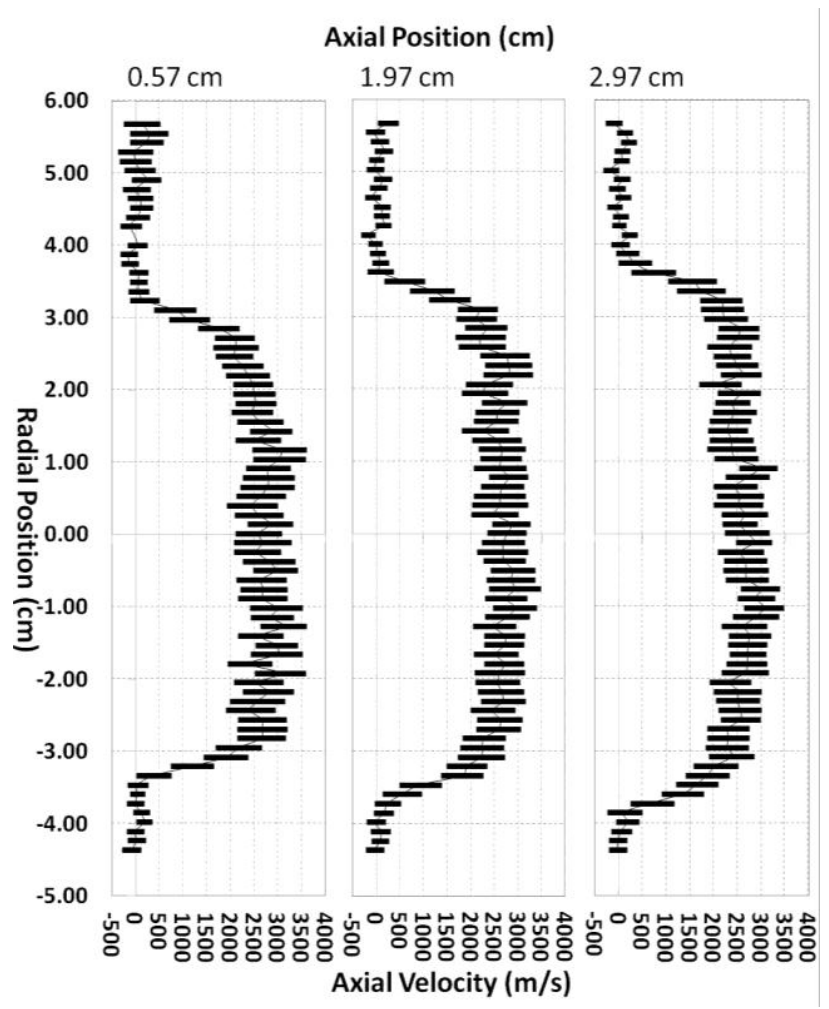

(b)

Fig. 15. Axial (a) average and (b) single-shot velocity profiles. 


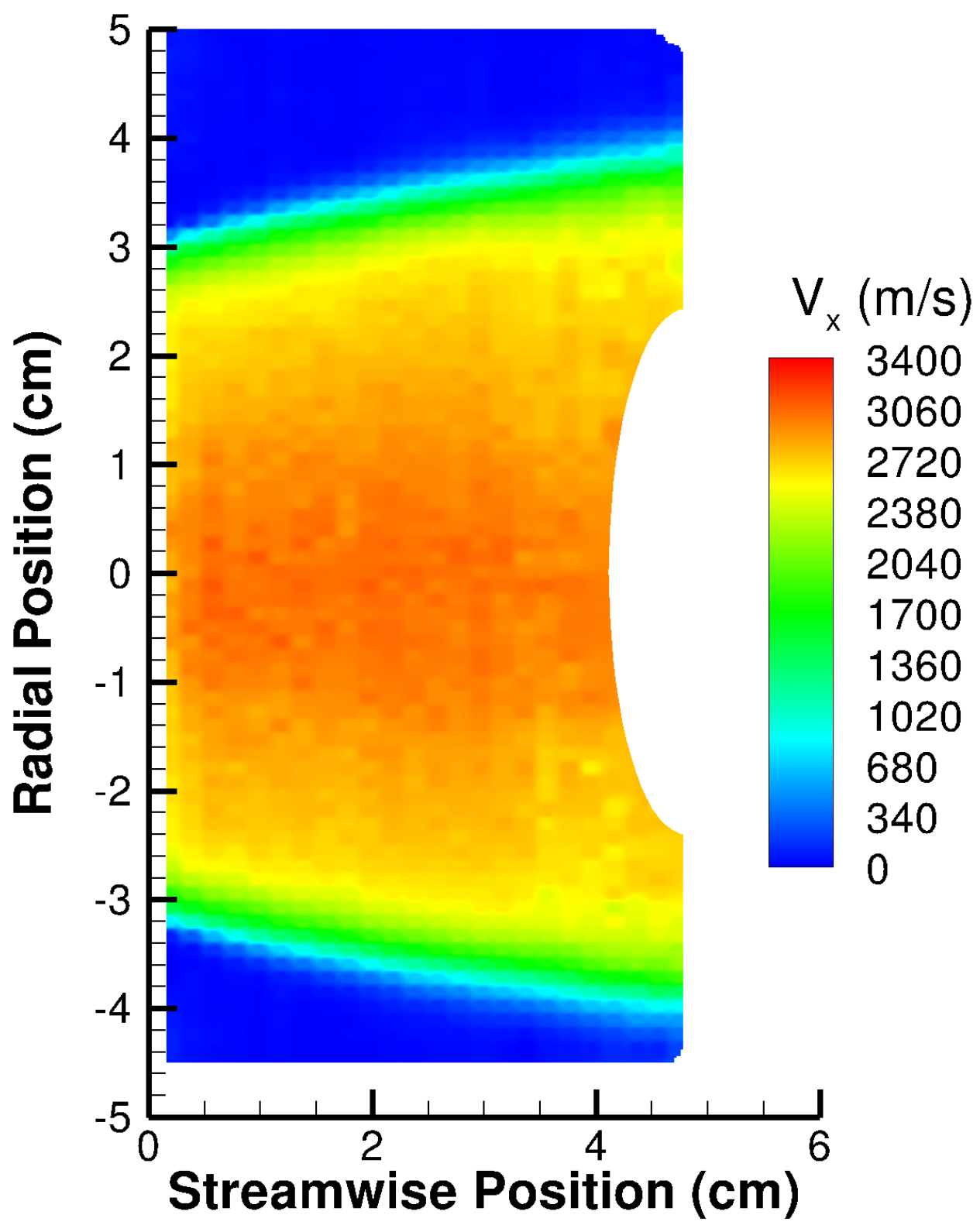

Fig. 16. Mean axial velocity field interpolated from MTV velocity profiles.

Figure 16 shows the axial velocity flowfield, interpolated over the entire region encompassing the measurements obtained along each tagged profile shown in Fig. 14a. The flow propagating down the centerline of the flow appears to have a nearly constant velocity, despite the fact that the nozzle is conical so the flow is diverging and continuing to expand.

C. Radial Velocity Analysis and Results 


\section{Analysis Method}

Using post processing software, ImageJ, a temporal distribution of the fluorescence signal was extracted from the image sequence at each spatial location. Since the laser scanning rate was synchronized to the camera system, a spectrum of the fluorescence signal versus wavelength could be obtained from the image data: each pixel in the flow resulting in an NO PLIF excitation spectrum. As stated in the Experimental Methods section, acquiring the data for a full wavelength scan at a given condition took approximately one minute. Figure 17 shows three typical spectra, obtained from three $4 \times 4$ binned regions located $4 \mathrm{~cm}$ downstream of the nozzle exit. ( $P 1$ is a region outside the core flow; $P 2$ is a region of large positive radial velocity, and $P 3$ is a region of large negative radial velocity. Figure 20a shows the location of regions $P 1, P 2$, and $P 3$ relative to the overall flowfield.) In Fig. 17, scatter points correspond to experiment and solid lines indicate a Gaussian curve fit. The locations from which spectra are extracted are depicted as points in Fig. 20a: $\mathrm{P} 1(\mathrm{x}=4 \mathrm{~cm}, \mathrm{y}=4.4 \mathrm{~cm}), \mathrm{P} 2(\mathrm{x}=4 \mathrm{~cm}, \mathrm{y}=2.8 \mathrm{~cm}), \mathrm{P} 3(\mathrm{x}=4 \mathrm{~cm}, \mathrm{y}=-2.8 \mathrm{~cm})$.

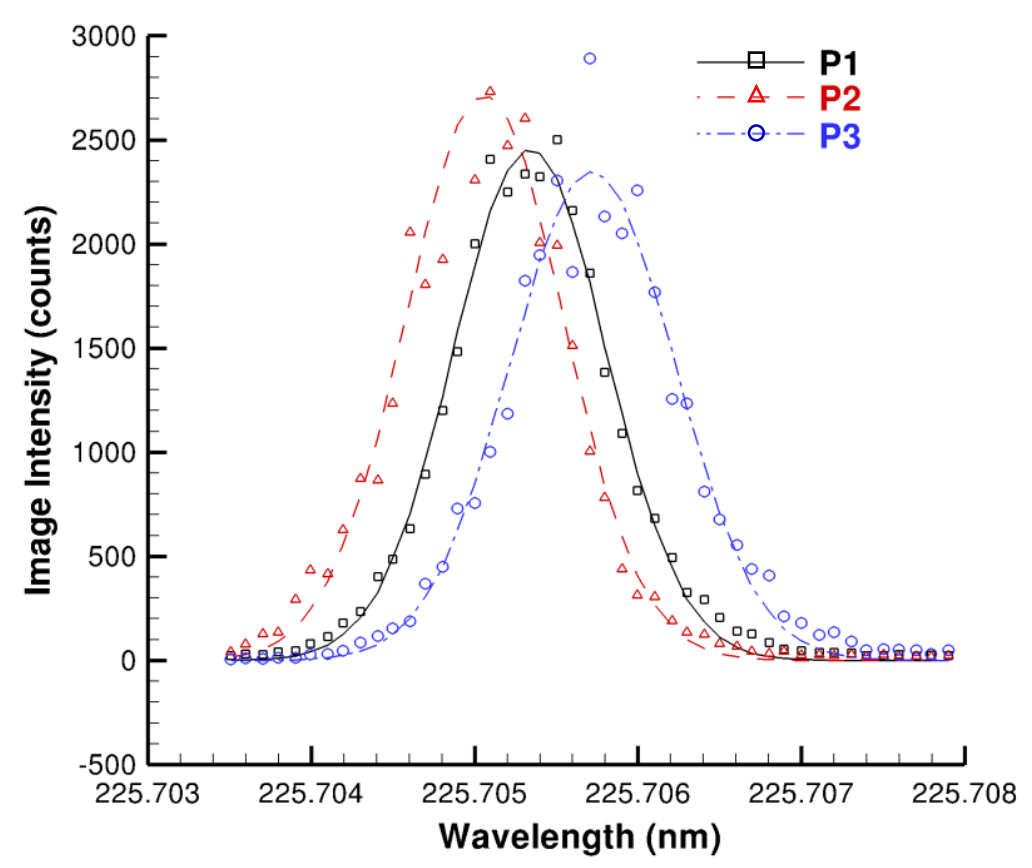

Fig. 17. Three typical spectra obtained from various locations in the flow. Symbols are experimental data and lines are Gaussian curve fits

The equation for the Gaussian curve fit shown in Fig. 17 is defined as:

$$
N_{p}=A e^{\left(\frac{-4 \ln (2)\left(\lambda-\lambda_{c}\right)^{2}}{\Delta \lambda^{2}}\right)}
$$


where $N_{p}, A, \lambda_{c}$, and $\Delta \lambda$ are the signal level, signal coefficient, transition center wavelength, and the apparent transition linewidth, respectively. The error, $E$, between the experimental data, $N_{p, \exp }$, and $N_{p}$ is defined as:

$$
E=\sum_{\lambda}^{\lambda_{\max }}\left(N_{p, \exp }-N_{p}\right)^{2}
$$

By differentiating Eq. (7) with respect to $A, \lambda_{c}$, and $\Delta \lambda$ it was possible to minimize the total error using the iterative Newton-Raphson method:

$$
\begin{gathered}
A_{i+1}=A_{i}-\frac{\partial E / \partial A}{\partial^{2} E / \partial A^{2}} \\
\lambda_{c, i+1}=\lambda_{c, i}-\frac{\partial E / \partial \lambda_{c}}{\partial^{2} E / \partial \lambda_{c}^{2}} \\
\Delta \lambda_{j+1}=\Delta \lambda_{j}-\frac{\partial E / \partial \Delta \lambda}{\partial^{2} E / \partial \Delta \lambda^{2}}
\end{gathered}
$$

To verify that each fit variable was properly optimized, the algorithm monitored the residual error between old and corrected values. If the calculated error for each fit parameter was less than the specified tolerance of $10^{-10}$, the optimization process would stop and the algorithm would proceed to the next spatial location. If the error exceeded the specified tolerance, the total number of iterations would double and an updated residual error would subsequently be calculated. For most regions of the flow, the optimization process would complete after 500 iterations. In regions of low signal-to-noise, the algorithm would require substantially more iterations to complete the process. If an upper limit of 1,000,000 iterations was reached at a single location, the algorithm would discard that portion of the experimental data. The discarding of measurements usually occurred in regions of very low signal-to-noise ratio or regions outside of the laser sheet interrogation volume (shadow regions or at the sample). By filtering out regions with low signal (i.e. < 25 counts) prior to optimization, faster processing was achieved.

After obtaining the values of $\lambda_{c}$ and $\Delta \lambda$ it is possible to calculate quantitative values of radial velocity. Since the laser sheet was projected normal to the axial hypersonic flow produced by the facility, any radial component of velocity will cause an apparent shift in the transition center frequency, $v_{c}$, relative to the laser center frequency, $v_{L}$. This shift is termed the Doppler shift and is defined as:

$$
\Delta v_{D S}=v_{c}-v_{L}
$$


The wavelength, $\lambda$, and Doppler shift in wavelength, $\Delta \lambda_{D S}$, are related to the spatial frequency, $v$ (which is the frequency in $\mathrm{Hz}$ divided by the speed of light in $\mathrm{cm} / \mathrm{s}$ ), and the Doppler shift in spatial frequency, $\Delta v_{D S}$, through the following equations:

$$
\begin{gathered}
v\left[\mathrm{~cm}^{-1}\right]=\frac{10^{7}\left[\mathrm{~cm}^{-1} \mathrm{~nm}\right]}{\lambda[\mathrm{nm}]} \\
\Delta v_{D S}\left[\mathrm{~cm}^{-1}\right]=\frac{-10^{7}\left[\mathrm{~cm}^{-1} \mathrm{~nm}\right] \Delta \lambda_{D S}[\mathrm{~nm}]}{\lambda^{2}\left[\mathrm{~nm}^{2}\right]}
\end{gathered}
$$

The Doppler shift is related to the component of radial velocity, $V_{r}$, and the speed of light, $c$, by:

$$
V_{r}=\frac{c \Delta v_{D S}}{v_{L}}
$$

The measured shifts in the excitation spectra were assumed to be due entirely to the Doppler shift, thereby ignoring the collisional shift (also known as pressure shift), which is significant at higher pressures. Errors resulting from this assumption are considered below. Also, in the analysis, symmetry was invoked only to determine where the centerline frequency reference was for stationary molecules. By symmetry, we assumed that the radial flow velocity in the outermost regions of the flow were equal in magnitude but opposite in sign. However, the Doppler shift was observed to be equal in these two regions. Thus the velocity in both of these regions must be zero, providing a convenient reference for zero Doppler shift.

\section{Uncertainty Analysis}

The main sources of random error are due to randomness in the data and low signal-to-noise ratio. As the tolerance in the optimization algorithm was set to $10^{-10}$, the corresponding uncertainty in the velocity is approximately $\pm 10^{-5} \mathrm{~m} / \mathrm{s}$, which is negligible. The uncertainty due to low signal-to-noise is demonstrated by looking at the effect of binning (Fig. 18). The effect of binning is to increase the total signal-to-noise ratio, but also to decrease the spatial resolution of the velocity measurement. 


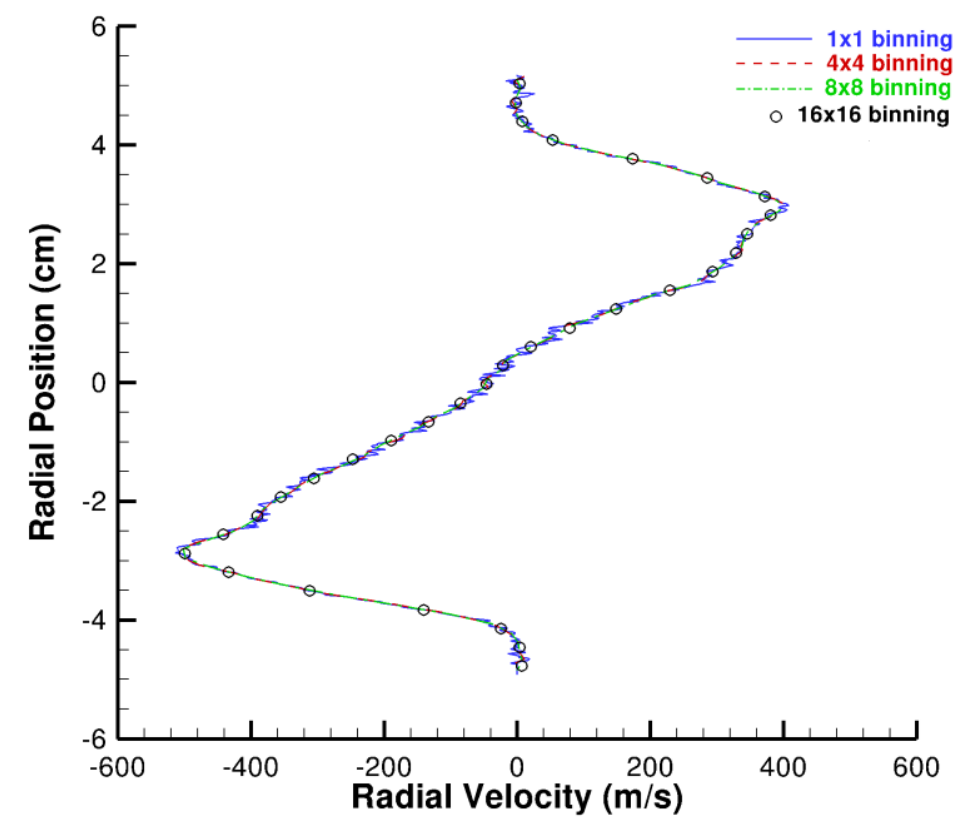

Fig. 18. Effect of binning on the radial distribution of radial velocity (10.8 MJ/kg Mars, Run 114).

It was determined that a $4 \mathrm{X} 4$ binning provided adequate spatial resolution to capture small scale structures and also provided sufficient smoothing to reduce random noise observed in the unbinned data of Fig. 18. Regions in the flow at the centerline and near the top and bottom wall surfaces have a near zero radial velocity component. By analyzing the fluctuation in velocity in those regions, an estimate for the uncertainty due to random noise and flow fluctuations could be determined. Near the top and bottom wall surfaces, the uncertainty was $\pm 16.8 \mathrm{~m} / \mathrm{s}$ (95\% confidence). A more conservative estimate of the error due to random noise and flow fluctuations could be determined from analyzing the variation in radial velocity near the flow centerline. This uncertainty was $\pm 26 \mathrm{~m} / \mathrm{s}$.

Sources of systematic error in the measurement include laser beam attenuation, wavelength linearity of laser, and collisional shift. Due to Beer's law of absorption, the laser intensity decreases approximately exponentially as the laser passes through the flow. The black curve in Fig. 19a shows the measured radial distribution of signal level $(A)$ at a streamwise position of $4 \mathrm{~cm}$ downstream of the nozzle exit. By assuming that the absorption coefficient is constant along the path of the light and by imposing symmetry on the fluorescence intensity profile, it is possible to calculate a radial profile of laser intensity and fit it with an exponential function (green curve in Fig. 19a). Correcting the measured fluorescence profile (black curve) by the attenuated laser beam profile (green curve) recovers a nearly symmetric fluorescence profile (red curve). The attenuation in laser intensity will be more 
significant near the center of the absorption transition. Doppler shifted spectra near the bottom of the image will therefore have a different laser intensity that varies depending on the Doppler shift (and resulting absorption) of the gas above. This error is simulated in Fig 19b. After applying a simulated laser intensity profile to the experimental data and re-fitting the shifted spectra (shown as dashed lines in Fig 19b), a systematic error in $\lambda_{\mathrm{c}}$ can be calculated (see red and blue curves in Fig. 19b). Laser beam attenuation acts to artificially amplify $\Delta v_{D}$ in regions of high absorption and large radial velocity, which results in an over prediction in the magnitude of radial velocity. The maximum uncertainty due to laser beam attenuation was measured to be $\pm 56 \mathrm{~m} / \mathrm{s}$.

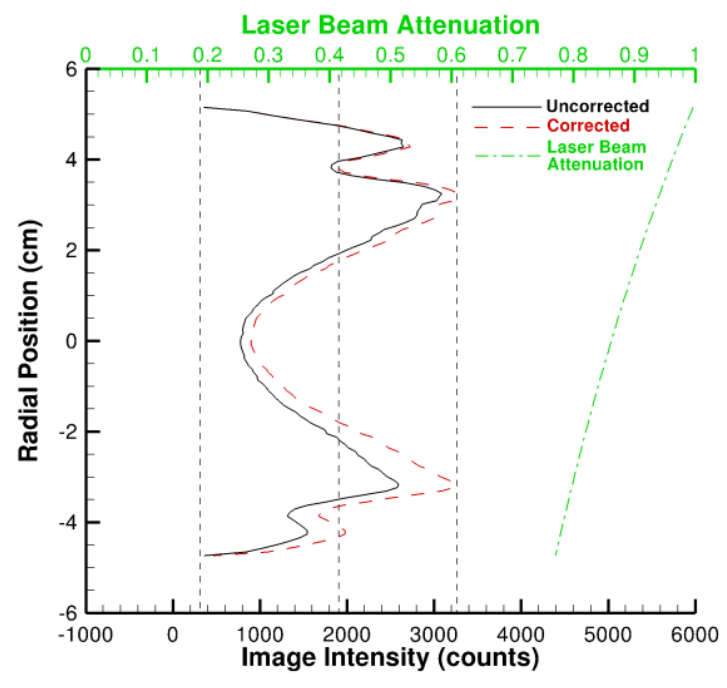

(a)

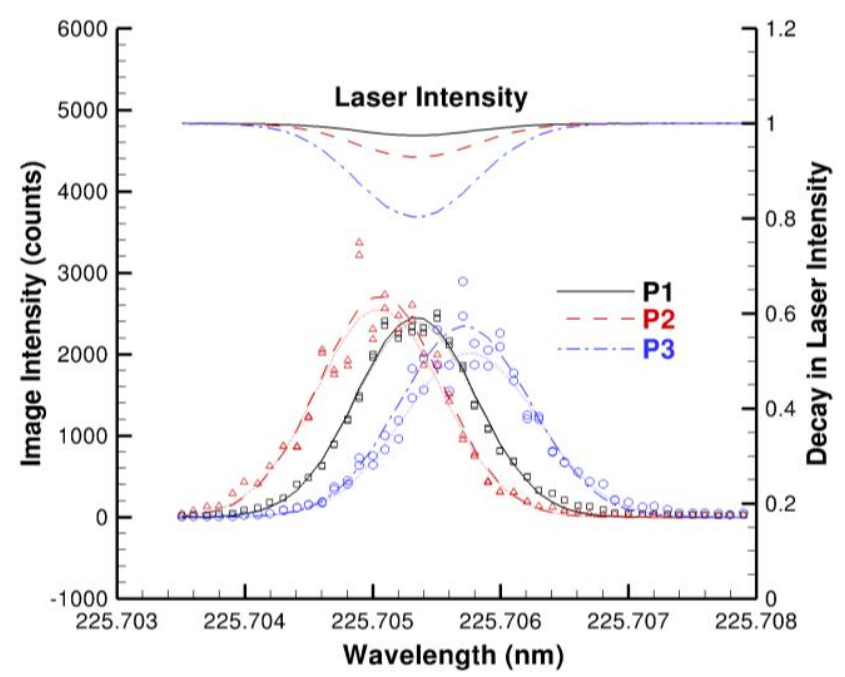

(b)

Fig. 19. Effect of laser beam attenuation on the measurement of Doppler shift.

There is also an uncertainty in the radial velocity measurement due to non-linearity in the laser scanning drive. Such an error would be caused by the laser's drive software not indicating the actual change in wavelength (absolute wavelength inaccuracy does not lead to an error in the current measurement). Based on the scan linearity measurements obtained from the manufacturer, a worst case error of approximately $\pm 0.0045 \mathrm{~cm}^{-1}$ in $\Delta v_{D}$ per wave number scanned was measured. This is equivalent to a systematic error in radial velocity of approximately $\pm 0.009\left(V_{r}\right)$. Thus, the maximum error in velocity due to non-linearity of the laser scanning is $\pm 4.5 \mathrm{~m} / \mathrm{s}$.

The collisional shift also contributes to a systematic error in radial velocity measurements. Although this is a chemically reacting flow for which a perfect gas analysis does not strictly apply, such an analysis can be useful for estimating static conditions in the jet for the purposes of this uncertainty analysis. For an isentropically expanded Mach 5 jet having a stagnation pressure (arc pressure) of $1.1 \mathrm{~atm}$, the pressure shift at the nozzle exit is 
approximately $0.0016 \mathrm{~cm}^{-1}$, which corresponds to a systematic error of just $1 \mathrm{~m} / \mathrm{s}$. This error was partially mitigated by the method by which the center wavelength, $\lambda_{\mathrm{L}}$, was calculated. Since $\lambda_{\mathrm{L}}$ is calculated from regions in the flow without a radial velocity component, any pressure shift resulting from a large uniform pressure field would be accounted for.

In summary, the total uncertainty in the flow velocity depends somewhat on the spatial location. The maximum velocity uncertainty measured in the flow is $\pm 62 \mathrm{~m} / \mathrm{s}$, which includes both random and systematic errors. This error is dominated by the laser beam attenuation. Table 2 shows the contribution of errors at three representative locations in the flow. In future experiments, weaker NO transitions could be probed to minimize the error caused by absorption.

Table 2 Contribution of random and systematic errors at three positions (P1-P3 located at $x=\mathbf{4}$ cm)

\begin{tabular}{lccc}
\hline \multicolumn{1}{c}{ Uncertainty Type } & $\mathrm{P} 1(\mathrm{y}=4.4 \mathrm{~cm})$ & $\mathrm{P} 2(\mathrm{y}=2.8 \mathrm{~cm})$ & $\mathrm{P} 3(\mathrm{y}=-2.8 \mathrm{~cm})$ \\
\hline Random Noise $(\mathrm{m} / \mathrm{s})$ & 25.9 & 25.9 & 25.9 \\
Laser Beam Attenuation $(\mathrm{m} / \mathrm{s})$ & 0.1 & 14.6 & 55.9 \\
Laser Non-Linearity $(\mathrm{m} / \mathrm{s})$ & 0.0 & 3.0 & 4.5 \\
Collisional Shift $(\mathrm{m} / \mathrm{s})$ & 1.1 & 1.1 & 1.1 \\
\hline Total Uncertainty $(\mathrm{m} / \mathrm{s})$ & 25.9 & 29.9 & 61.8 \\
\hline \hline
\end{tabular}

\section{Radial Velocity Results}

Figure 20a shows a vector plot for the $6.5 \mathrm{MJ} / \mathrm{kg}$ Earth condition (Run 114) overlaid with contours of radial velocity. The streamwise component of velocity was determined from the MTV technique (Run 157). Fig. 20b shows distributions of radial velocity at four different streamwise locations labeled L1-L4. The position of lines L1L4 are shown as white dashed lines in Fig. 20a. The white points, labeled P1-P3 are the positions where the spectra shown in Fig. 17 were obtained. Due to a varying systematic error, the magnitude of the error bars in Fig. 20b also vary with radial and streamwise position. Due to the systematic nature of these errors, the error bars are also positioned asymmetrically to reflect the direction that laser beam attenuation and laser wavelength non-linearity affect radial velocity. The regions of Fig. 20a not containing measurements correspond to regions of the flow where the optimization algorithm discarded data due to low signal. 


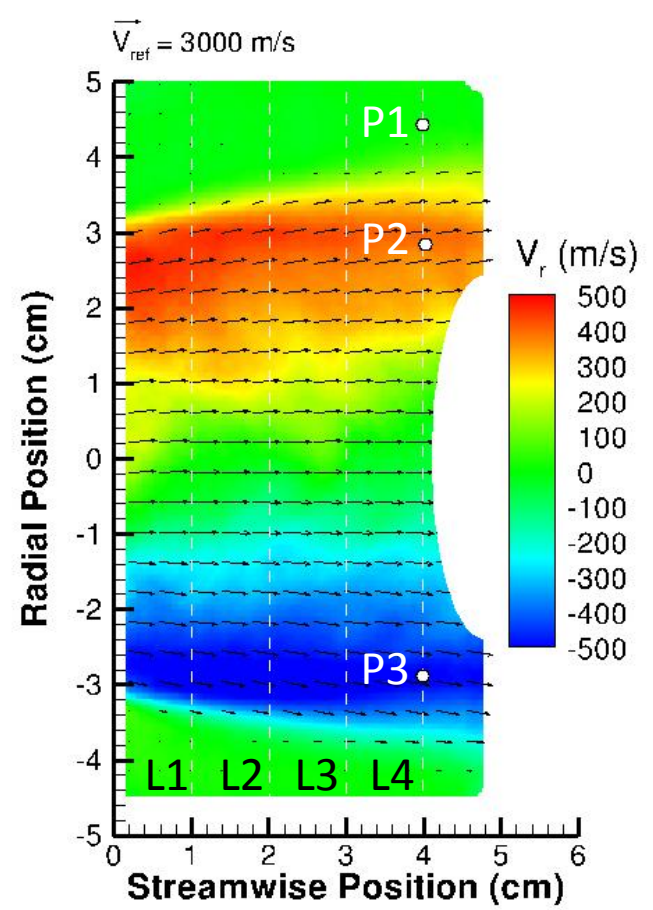

(a)

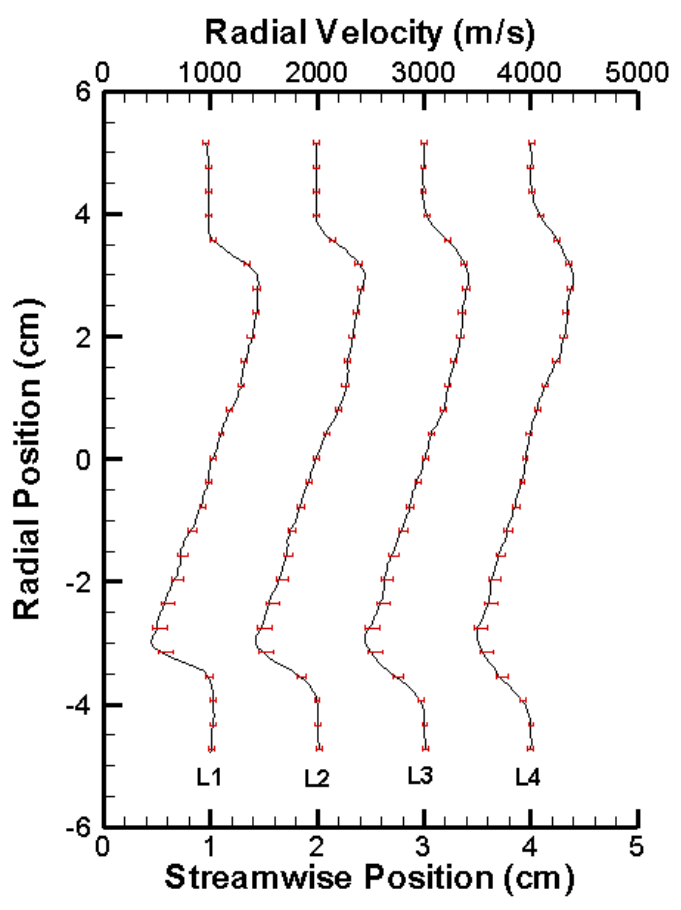

(b)

Fig. 20. Map (a) and profile (b) distributions of radial velocity for $6.5 \mathrm{MJ} / \mathrm{kg}$ Earth condition (Run 114).

Figure 21 shows a comparison between the velocity field measured from the $6.5 \mathrm{MJ} / \mathrm{kg}$ Earth condition (Run 114 and Run 157; black vectors) to a theoretical velocity field (red vectors) assuming a perfectly expanded jet with a nozzle half-angle of 8 degrees. To construct the field, a point source was located upstream of the nozzle, the location of which was determined by projecting the nozzle walls back to a single point. In the theoretical velocity field, the total velocity magnitude was considered constant downstream of the nozzle at a value of $2994 \mathrm{~m} / \mathrm{s}$, which corresponds to the average centerline streamwise velocity in the experimental data. Although qualitative, there is close agreement between the two cases, which confirms the essentially point-source nature of the flow produced by the facility and gives overall confidence in both the Doppler-shift based velocimetry and MTV techniques. 


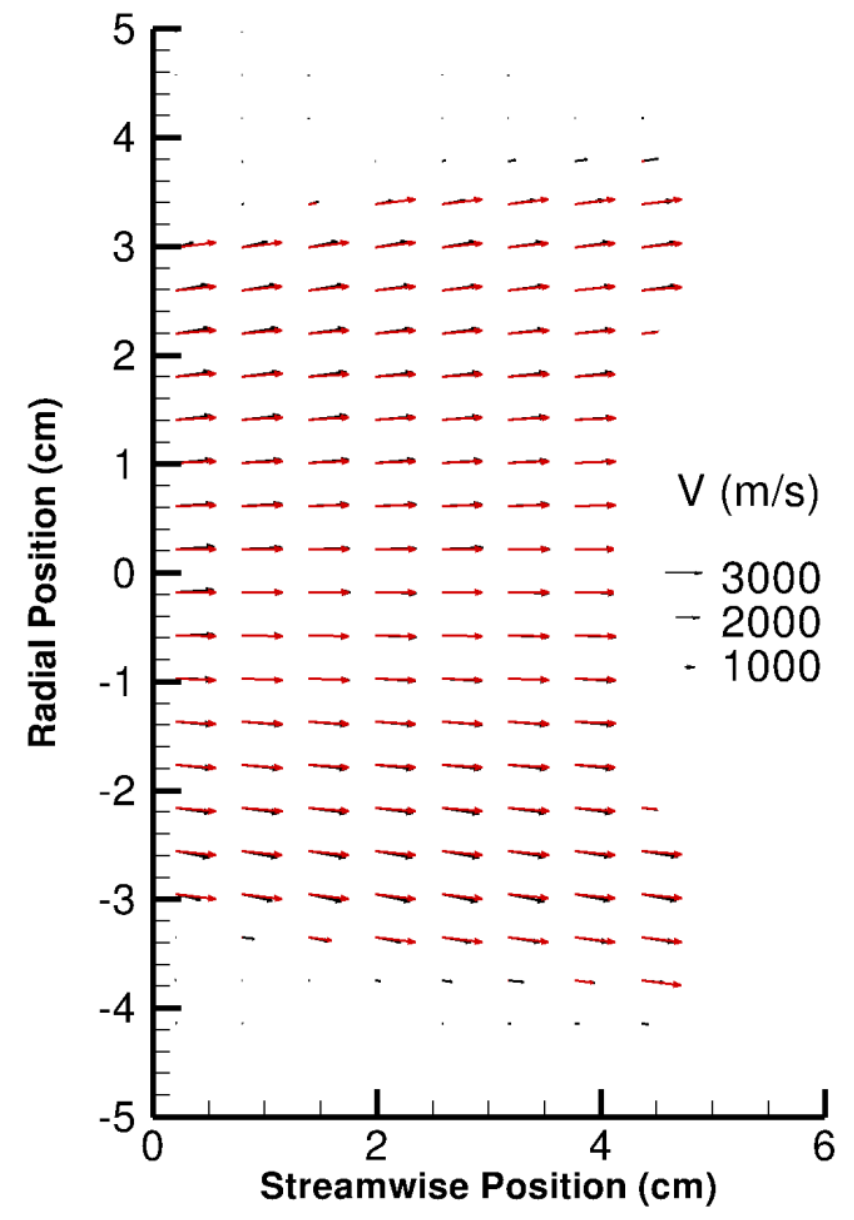

\section{Fig. 21. Comparison of an experimental velocity field (black vectors) to an ideal diverging point-source velocity field (red vectors).}

Figure 22 shows a comparison of maps of measured radial velocity for facility runs corresponding to the 6.5 MJ/kg Earth condition (Run 114) and the 10.8 MJ/kg Mars condition (Run 85). Although the overall magnitudes in radial velocity are similar in the top portion of the image, the magnitude of radial velocity measured for the 6.5 $\mathrm{MJ} / \mathrm{kg}$ Earth condition is $25 \%$ larger in the lower portion of the image. For the $10.8 \mathrm{MJ} / \mathrm{kg}$ Mars condition, the magnitudes in radial velocity are similar for both the top and bottom portions of the image. This may indicate that the systematic error in radial velocity due to laser beam attenuation is larger for the $6.5 \mathrm{MJ} / \mathrm{kg}$ Earth condition compared to the $10.8 \mathrm{MJ} / \mathrm{kg}$ Mars condition. 

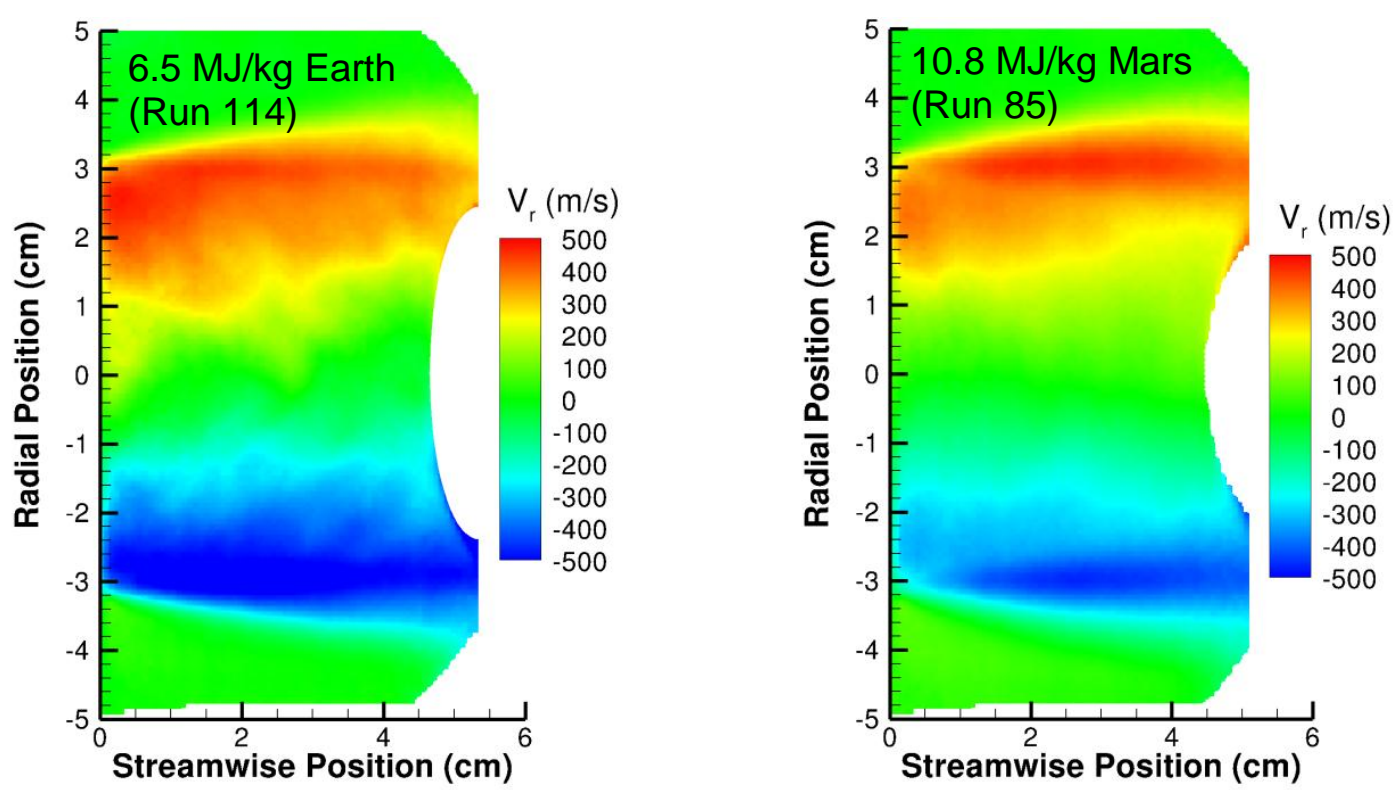

Fig. 22. Comparison of radial distribution of velocity shown as a contour plot for the $6.5 \mathrm{MJ} / \mathrm{kg}$ Earth condition (Run 114) and 10.8 MJ/kg Earth condition (Run 85).

\section{Free Stream Static Temperature Estimation}

From measurements of the spectral width, $\Delta v_{\mathrm{DB}}$, of the Doppler-broadened Gaussian distribution such as shown in Fig. 17, it is possible to extract the translational temperature, $T$, from the following relationship:

$$
\Delta v_{D B}=v_{L} \sqrt{\frac{8 k(\ln (2)) N_{A} T}{\left(\mathrm{MW}_{\mathrm{NO}}\right) c^{2}}}
$$

where $k, N_{A}, c, \mathrm{MW}_{\mathrm{NO}}$ are the Boltzmann constant, Avogadro's number, speed of light, and the molecular weight of NO, respectively. The average translational temperature in the core of the jet at the nozzle exit was measured to be $\sim 1,300 \mathrm{~K}\left(\sim 1,900{ }^{\circ} \mathrm{F}\right)$ for the $6.5 \mathrm{MJ} / \mathrm{kg}$ Earth condition and 1,600 $\mathrm{K}\left(2,400{ }^{\circ} \mathrm{F}\right)$ for the $10.8 \mathrm{MJ} / \mathrm{kg}$ Mars condition. When determining $\Delta v_{\mathrm{DB}}$ in the fitting process, collisional broadening was neglected. Additionally, we assumed that the laser's linewidth of $0.07 \mathrm{~cm}^{-1}$ added in quadrature with $\Delta v_{\mathrm{DB}}$ to fit the measured linewidth. The effect of laser beam absorption and saturation are systematic errors that both artificially broaden the transition. Therefore, the actual translational temperature is likely cooler than measured here if saturation and absorption are significant. Thus, the above reported temperature is an estimation of the upper limit of the average free stream static translational temperature. 


\section{Conclusion}

NO PLIF has been successfully applied to the HYMETS facility at NASA Langley Research Center for the first time. Flow visualization, velocity measurements, and a calculation of an upper bound on the flow temperature have helped to characterize previously uncertain flow parameters by providing both qualitative and quantitative temporally and spatially resolved information about the arcjet flow. The techniques demonstrated in this paper are expected to be applicable in arc-jet flows having enthalpies of less than $10 \mathrm{MJ} / \mathrm{kg}$, where most images exhibit strong fluorescence. At higher enthalpies, O-atom and N-atom LIF can be used to determine flow properties, although these are point measurements as opposed to planar measurements.

\section{Acknowledgments}

The authors wish to thank Amy Brewer for her help in running the arcjet facility, as well as summer students Jeff Wheeler and Ethan Brewer for their contributions in acquiring the data presented in this paper. This work was funded in part by NASA's Fundamental Aeronautics Program, Hypersonics Project, Experimental Capabilities Discipline.

\section{References}

[1] Splinter, S.C., Bey, K.S., Gragg, J.G., and Brewer, A.R., "Comparative Measurements of Earth and Martian Entry Environments in the NASA Langley HYMETS Facility," $49^{\text {th }}$ AIAA Aerospace Sciences Meeting, Orlando, FL, AIAA Paper 2011-1014, Jan. 2011.

[2] Zhang, F.-Y., Komurasaki, K., Iida, T., and Fujiwara, T., "Diagnostics of an Argon Arcjet Plume with a Diode Laser,” Applied Optics, Vol. 38, No. 9, 20 March 1999, pp. 1814-1822.

doi: 10.1364/AO.38.001814

[3] Grinstead, J.H., Driver, D.M., and Raiche, G.A., "Radial Profiles of Arcjet Flow Porperties Measured with Laser-Induced Fluorescence of Atomic Nitrogen," $41^{\text {st }}$ Aerospace Sciences Metting, Reno, NV, AIAA Paper 2003-0400, Jan. 2003.

[4] Grinstead, J.H., Harris, C.L., Yeung, Dickson, S., Gregg P., Porter, B.J., Graube, P., and Greenberg, R.B., "NextGeneration Laser-Induced Fluorescence Diagnostic Systems for NASA Arc Jet Facilities," $47^{\text {th }}$ AIAA Aerospace Sciences, Orlando, FL, AIAA Paper 2009-1521, Jan. 2009. 
[5] Takayanagi, H., Mizuno, M., Fujii, K., Suzuki, T., and Fujita, K., “Arc Heated Wind Tunnel Flow Diagnostics using Laser-Induced Fluorescence of Atomic Species," $47^{\text {th }}$ AIAA Aerospace Sciences, Orlando, FL, AIAA Paper 2009-1449, Jan. 2009.

[6] Del Vecchio, A., Palumbo, G., Koch, U., and Gulhan, A., “Temperature Measurements by Laser-Induced Fluorescence Spectroscopy in Nonequilibrium High-Enthalpy Flow," Journal of Thermophysics and Heat Transfer, Vol. 14, No. 2, April-June 2000, pp. 216-224.

doi: $10.2514 / 2.6511$

[7] Mizuno, M., Ito, T., Ishida, K., and Nagai, J., "Laser Induced Fluorescence of Nitric Oxide and Atomic Oxygen in an Arc Heated Wind Tunnel," 39 ${ }^{\text {th }}$ AIAA Thermophysics Conference, Miami, FL, AIAA Paper 2007-4405, Jun. 2007.

[8] Scott, C.D., "Survey of Measurements of Flow Properties in Arcjets," Journal of Thermophysics and Heat Transfer, Vol. 7, No. 1, Jan.-March 1993, pp. 9-24.

doi: $10.2514 / 3.11563$

[9] Arepalli, S., Yuen, E.H., and Scott, C.D., “Application of Laser Induced Fluorescence for Flow Diagnostics in Arc Jets," AIAA/ASME $5^{\text {th }}$ Joint Thermophysics and Heat Transfer Conference, Seattle, WA, AIAA Paper 901763, Jun. 1990.

[10] Bathel, B.F., Danehy, P.M., Inman, J.A., Jones, S.B., Ivey, C.B., and Goyne, C.P., "Multiple Velocity Profile Measurements in Hypersonic Flows Using Sequentially-Imaged Fluorescence Tagging," 48 ${ }^{\text {th }}$ AIAA Aerospace Sciences, Orlando, FL, AIAA Paper 2010-1404, Jan., 2010.

[11] Bathel, B.F., Danehy, P.M., Inman, J.A., Jones, S.B., Ivey, C.B., and Goyne, C.P., "Velocity Profile Measurements in Hypersonic Flows Using Sequentially Imaged Fluorescence-Base Molecular Tagging,” AIAA Journal, Vol. 49, No. 9, Sept. 2011, pp. 1883-1896.

doi: $10.2514 / 1 . J 050722$

[12] Hiller, B., McDaniel, J.C., Rea, E.C., and Hanson, R.K., "Laser-induced fluorescence technique for velocity field measurements in subsonic gas flows," Optics Letters, Vol. 8, No. 9, Sept., 1983, pp. 474-476. doi: 10.1364/OL.8.000474

[13] McDaniel, J.C., Hiller, B., and Hanson, R.K., "Simultaneous multiple-point velocity measurements using laser induced iodine fluorescence," Optics Letters, Vol. 8, No. 1, Jan., 1983, pp. 51-53. 
doi: 10.1364/OL.8.000051

[14] Palmer, J.L. and Hanson, R.K., "Single-shot velocimetry using planar laser-induced fluorescence imaging of nitric oxide," AIAA/SAE/ASME/ASEE $29^{\text {th }}$ Joint Propulsion Conference, Monterey, CA, AIAA Paper 93-2020, Jun 1993.

[15] Danehy, P.M., Mere, P., Gaston, M.J., O’Bryne, S., Palma, P.C., and Houwing, A.F.P., "Fluorescence velocimetry of the hypersonic, separated flow over a cone.” AIAA Journal, Vol. 39, No. 7, Jul., 2001, pp. 13201328.

doi: $10.2514 / 2.1450$

[16] Hruschka, R., O’Byrne, S., and Kleine, H., “Two-component Doppler-shift fluorescence velocimetry applied to a generic planetary entry probe model," Experiments in Fluids, Vol. 48, No. 6, Jun. 2010, pp. 1109-1120. doi: 10.1007/s00348-009-0794-3

[17] Hiller, B., and Hanson, R.K., "Simultaneous planar measurements of velocity and pressure fields in gas flows using laser induced fluorescence", Applied Optics, Vol. 27, No. 1, Jan., 1988, pp. 33-48. doi: 10.1364/AO.27.000033

[18] Paul, P.H., Lee, M.P., Hanson, R.K., "Molecular velocity imaging of supersonic flows using pulsed planar laser induced fluorescence of NO," Optics Letters, Vol. 14, No. 9, May 1989, pp. 417-419. doi: 10.1364/OL.14.000417

[19] Bathel, B.F., Danehy, P.M., Inman, J.A., Watkins, A.N., Jones, S.B., Lipford, W.E., Goodman, K.Z., Ivey, C.B., and Goyne, C.P., "Hypersonic Laminar Boundary Layer Velocimetry with Discrete Roughness on a Flat Plate," 40 $0^{\text {th }}$ Fluid Dynamics Conference, Chicago, IL, AIAA Paper 2010-4998, Jun., 2010.

[20] Danehy, P.M., Alderfer, D.W., Inman, J.A., Berger, K.T., Buck, G.M., and Schwartz, R.J., "Fluorescence Imaging and Streakline Visualization of Hypersonic Flow over Rapid Prototype Wind-Tunnel Models,” Proc. IMechE, Part G: Journal of Aerospace Engineering, Vol. 222, No. 5, 2008, pp. 637-651. doi: 10.1243/09544100JAERO295

[21] Sorzano, C.O.S., Thévenaz, P., and Unser, M., "Elastic Registration of Biological Images Using Vector-Spline Regularization," IEEE Transactions on Biomedical Engineering, Vol. 52, No. 4, April, 2005, pp. 652-663. doi: 10.1109/TBME.2005.844030 